\title{
APPLICATION OF STYLOMETRY TO DARK WEB FORUM USER IDENTIFICATION
}

HO THANH NGHIA

School of Computer Science and Engineering

\author{
A thesis submitted to the Nanyang Technological University \\ in partial fulfilment of the requirement for the degree of \\ Master of Engineering
}


NANYANG TEChNOLOGICAL University

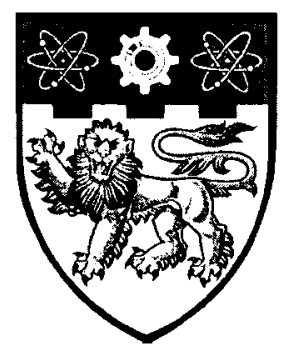

\title{
Application of Stylometry to Dark Web Forum User Identification
}

\author{
Master Thesis \\ By \\ Ho Thanh Nghia \\ Supervisor: Dr. Ng Wee Keong
}

School of Computer Science and Engineering

A thesis submitted to the Nanyang Technological University

in fulfillment of the requirement for the degree of Master of Engineering

January, 2017 


\section{Acknowledgments}

I would like to express my decpest gratitude to my supcrvisor in charge, Dr. Ng Wee Keong, for his continuous encouragement as well as his invaluable guidance on my research. As a supervisor, he has taught me a lot of research skills and essential knowledge that has significantly inspired and benefited me in my academic career. Out of academic, I also consider him a life mentor who is always willing to advise me on many struggles and issues.

I would also like to thank Liu Fang: Quach Vinh Thanh and Huynh Ngoc Anh for their valuable suggestions, which have greatly assisted me in completing this report.

Last but not least, I am extremely grateful to my parents for their encouragement and support during my hard times. Without them, this work definitely could not have been accomplished. 


\section{Contents}

Acknowledgments .....................

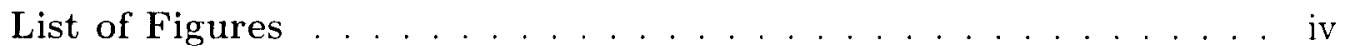

List of Tables . . . . . . . . . . . . . . . . . v

Summary ........................... vi

1 Introduction $\quad 1$

2 Literature Survey $\quad 5$

2.1 Authorship analysis . . . . . . . . . . . . . . 6

2.2 Dark Web forums . . . . . . . . . . . . . . . 7

2.3 Authorship attribution in online messaging . . . . . . . . . . 11

2.4 Writing-Style Features . . . . . . . . . . . . . . . . 13

2.5 Attribution Techniques .................... 14

2.6 Relevant Datasets . . . . . . . . . . . . . . . . . . 16

$\begin{array}{lll}3 & \text { Research Problems and Methods } & 18\end{array}$

3.1 Uscr Identity Matching across Dark Web . . . . . . . . . . . . . 18

3.2 Research Objective . . . . . . . . . . . . . . . . . . . . . . . 19

3.3 Framework. . . . . . . . . . . . . . . 19

3.3 .1 Message Collection . . . . . . . . . . . . . . . 20

3.3 .2 Message Filtering . . . . . . . . . . . . . 22

3.3 .3 Feature Extraction . . . . . . . . . . . . . . 25

3.3 .4 Model Generation . . . . . . . . . . . . . . 26

3.3 .5 Authorship Matching . . . . . . . . . . . . . . 26 
$\begin{array}{lll}4 & \text { Experiments } & 27\end{array}$

4.1 Validation Phase . . . . . . . . . . . . . . . . 29

4.2 Testing Phase . . . . . . . . . . . . . . . . . . 32

4.3 Feature Selection . . . . . . . . . . . . . 34

4.4 Classifier Generation . . . . . . . . . . . . . . 38

5 Conclusion 45

6 List of Authors Publications 47

6.1 Accepted Paper . . . . . . . . . . . . . . . . . . . . . 47 


\section{List of Figures}

2.1 The Internet layout (from http://www.deepwebtech.com) . . . . . . 8

2.2 TOR's workflow (from http://arstechnica.com) . . . . . . . . . 9

2.3 A typical Dark Web forurn structure . . . . . . . . . . . . . . . 10

2.4 Support Vector Machines (SVM) classification . . . . . . . . 16

3.1 Authorship matching process pipeline . . . . . . . . . . . . . 20

3.2 A sample user's post in a Dark Web forum . . . . . . . . . . . . 21

3.3 HTML to raw text data transformation using parser . . . . . . . . 22

3.4 A sample part of an author's document . . . . . . . . . . . 22

3.5 A sample conversation including PGP encryption's texts . . . . . . 23

4.1 Authorship matching task . . . . . . . . . . . . . 2 28

4.2 Validation experiment details ................... 31

4.3 Testing experiment details . . . . . . . . . . . . . . . 33

4.4 One-against-all SVM classification . . . . . . . . . . . . 40 


\section{List of Tables}

4.1 Dataset overview . . . . . . . . . . . . . . . . . . . . 29

4.2 Dataset matching users statistics . . . . . . . . . . . . 29

4.3 Experiment 1 dataset overview . . . . . . . . . . . . . 32

4.4 Experiment 2 dataset overvicw . . . . . . . . . . . . 34

4.5 Features vectors description . . . . . . . . . . . . . 37

4.6 Data representation differences between Jstylo and LibSVM . . . . . 39

4.7 Validation experiment result (linear kernel) . . . . . . . . . . . . 42

4.8 Validation expcriment result (polynomial kernel) . . . . . . . . . . 42

4.9 Validation experiment result (rbf kernel) . . . . . . . . . . . . 43

4.10 Validation experiments $F_{1}$ score $\ldots \ldots \ldots 43$

4.11 Test experiment result (linear kernel) . . . . . . . . . . . . . . . . 43

4.12 Test experiment result (polynomial kernel) . . . . . . . . . . . . 44

4.13 Test experiments $F_{1}$ score $\ldots \ldots \ldots \ldots . \ldots \ldots 4$

4.14 Experiment results using different classification methods in micro $F_{1}$. . 44

4.15 Experiment results using different classification methods in macro $F_{1}$. 44 


\section{Summary}

The aim of authorship identification is to recover the original author of anonymous writing by leveraging a host of features extracted from the writing. Computational stylometry is the study of writing style of typed natural language texts in order to solve the authorship identification problem. As communications across Internet resources are growing rapidly in recent years, especially in online criminal activities, authorship analysis of web-based messages is becoming increasingly important. The anonymous nature of online forums, especially Dark Web forums such as the well-known "Silk Road", makes them ideal enviromments for criminal discussions. The user only needs to create an account with an anonymous uscrname on one of the Dark Web forums and can start doing his illegal businesses. Ranging from government, security agencies to financial institutions, many parties are willing to trace the identities of the suspects through these online conversations. To this end, authorship analysis techniques have become increasingly relevant and crucial for exposing anonymous identities in cyber environments.

We consider the scenario where there are two accounts with the same username on two different Dark Web forums, $A$ and $B$. The user on forum $A$ carclessly cxposes his personal identities (name, location, phone number, etc.) in his posts and the user in forum $B$ commits certain illegal transactions. The interested parties want to determine whether these two accounts are associated to the same person. To achieve this purpose, we develop a statc-of-thc-art authorship matching method bascd on writing style analysis. The proposed technique is evaluated in a two-step validation process. Firstly, we evaluate the performance of the method using users' text data collected from the same forum. Secondly, when we are confident with the model, we carry out 
actual authorship matching on data gathered from different forums. Our experiment results show that the proposed framework can achiere good performance on identifying the correlations between Dark Web users based on their writings. However, there is still much to improve in order to utilize the proposed method for solving the real-world problems. We present a list of research challenges that should be addressed in future works before we can apply the technique in the actual practice. 


\section{等 Introduction}

Nowadays, cybercrimes have become common threats due to the fast growth of information technology (IT) in recent years. Generally, cybercrime is the act of exploiting Internet resources to commit illegal activities ranging from identity theft, drug salc, credit card stolen, child pornography, etc. It has been shown through past experiences that dealing with this crime wave is not a trivial task. The anonymous nature of the Internet and the ease to enter online criminal markets have greatly contributed to the dramatic increase in cybercrimes. For example, according to Singapore Police Force (SPF) in its annual crime report in 2015, online commercial crimes are the main factor in the $4 \%$ rise of overall crime in Singapore. The existence of Dark Web further enhances the anonymity of online criminals. Unlike the surface web, Dark Web can only be accessed using the TOR browser which provides a mechanism to hide surfing users' information from the Internet Scrvice Providers (ISP). As a result, law enforcement agencies have difficulty in gathering as much information about the criminals on the Dark Web as in the surface web. However, there is no such thing as a perfect crime. The crininals may mistakenly leave some trails on the Internet in various forms: texts, images, registered information, etc. On multiple occasions, we can extract valuable information which may help to reveal the identities of the criminals 
by examining these trails carefully. In this research, we mainly focus on the criminal text data in Dark Web forums. Suppose the scenario where a suspect mistakenly posts partial of his personal information in a forum's message. In addition, we can extract other useful information from another account with the same username on a different Dark Web forum. Being able to validate that the two accounts belong to the same person with high enough confidence allows us to combine various scattering pieces of information into a more concrete and advanced form of knowledge. Such knowledge will in turn leads to actionable insights which are very useful for bringing the criminals to justice. Thercfore, cross identity matching across different Dark Web forums needs to be performed.

As stated earlier, profiling users on Dark Web forums is challenging. The lack of media data (photos, videos) on Dark Web forums forces us to focus our research on the text data alone. The raw text data collected from Dark Web forums poses some difficulties for stylometry analysis as it contains a lot of noises. Behind the user's anonymity caused by the TOR network, the obfuscation of important messages on some Dark Web forums also makes the identity tracking process harder. On the surface web, all the public data are visible to everyone. In contrast, the usage of data encryption methods allows the users to hide sensitive contents in Dark Web. For example, drug sellers and buyers can utilize the Pretty Good Privacy (PGP) encryption technique to exchange shipping information secretly. PGP helps to transform the messages into meaningless blocks of texts, which can only be decrypted by the participated parties who own the key. In addition, underground forums also share some similarities with the forums on the surface web. The existences of spammed messages (from humans and computer bots) further amplify the confusions in the text analysis process. By definition, forum spams consist of posts that contain related or unrelated advertisements, links to malicious websites, trolling and abusive or otherwise unwanted information. Most of the spammed messages are copied from other sources and repeatedly posted. Hence, they contribute very little to the user's profiling task through stylometry. Therefore, a filtering pre-process on the data is required to improve the accuracy of the experiments. 
This thesis aims to apply the existing text stylometry techniques for author identification of uscrs in Dark Web forums. Specifically, this thesis would like to propose a method for linking and matching users across Dark Web forums based on their unique writing style. In this research, we applied the authorship matching method on a sample set of 10 active Dark Web forum users. We call this set as $A$. We defined the active users as the oncs who have many posts on a Dark Web forum, the total length of which should be larger than 20000 words. Another condition is that we can find at least one active account with the same username on another Dark Web forum. As a result, we can find another set of 10 users that we label as $B$. We then compare the writing similarity between different and equal-size chunks of post messages in set $A$ alone and between $A$ and $B$.

Our experiments assume that the users in the Dark Web forums do not aware of the probability of identity leak through their writing habits. As a result, they do not attempt to adjust or modify their own writing style to protect their anonymity. However, it has been shown that humans are capable of intentionally altering their writing style if they want [7]. We cannot guarantee that our current work can apply efficiently in case of conscious text modification. We really hope that our work can motivate other researches of authorship identification in Dark Web environment that can meet all the real-world challenges. We are pretty confident that the validity of the presented method can be enhanced over time as better techniques are developed. Last but not least, our work has implications for the authorship identification and matching of users based on their post messages that exist in multiple Dark Web forums and can be applied in other text corpuses around the world.

The rest of the thesis is organized as follows.

- In Chapter 2, we review the background knowledge on authorship analysis and Dark Web that is required to understand subsequent parts of the thesis. We review previous works which tackle the problem of authorship attribution. In addition, we also present the state-of-the-art techniques to identify authorship and the datasets as well as the features commonly used by other researchers in the evaluation of their algorithm. 
- In Chapter 3 of Research Problems and Methods, we formally introduce the aforementioned problem of authorship matching between users on multiple Dark Web and the research objectives of this thesis. We introduce our novel authorship attribution algorithm and present it in the form of a data processing pipeline which involves five sequential processes: message collection, message filtering, feature extraction, model generation and the actual author matching.

- In Chapter 4 of Experiments, we cvaluate our algorithm on a datasct collected from multiple Dark Web forums. This use case represents a pressing problem on computer security of criminal identification. We discuss the results of the experiments using our framework with different parameter settings.

- Finally, in Chapter 5 of Conclusion, we give a conclusion for this thesis and discuss a few potential future works. 


\section{6) 4. \\ Literature Survey}

Firstly, we are going to provide some background information on authorship analysis. As we are particularly interested in the information available in Dark Web forums, we also describe shortly the context for the emergence of Dark Web forums. There are many restrictions on the surface web that the criminals want to overcome in order to do illegal transactions safely. The surface web can log all the information of the users which can be analyzed by legal parties. Examples of user information that can be collected include IP address, login time, browsers used ctc. Based on those results, the identity of the involved partics can be revealed casily. Inexperienced criminals are often exposed to the investigators through some unconscious online behaviours. For example, a drug buyer used Google search engine to find some products but forget to logout from his personal Google account.

Sccondly, sellers do not want to set up online shops where law enforcements can easily determine, for instance, who registered that domain or where the site's IP address located in the real world. In addition, anonymous communications between users are preferred to avoid government surveillance. Therefore, the existence of Dark Web which guarantecs anonymity and provides an obscure sense of security is a good environment for outlaw transactions and communications. DarkWeb forums, 
which reside on Dark Web, are the places where users can come to discuss about illegal activities in an anonymous manner. As mentioned above, the infrastructure of Dark Web helps to prevent investigators, policemen and other law enforcements from tracking the user's information including IP address, physical location or other private identification. Therefore, the application of feasible authorship analysis on Dark Web forum's data can help interested parties to reveal useful information about the suspects which cannot be easily determined on the surface web.

\subsection{Authorship analysis}

It is a common perception that the documents written by different individuals possess rather different characteristics. Authorship analysis is the field of research which aims to characterize these differences via statistical and computational methods. There are multiple branches of authorship analysis including authorship identification which is our main research interest. By definition, authorship identification refers to the technique of searching for the most appropriate owner of an unclaimed document from a given set of known authors. Authorship identification is performed by comparing the stylistic features between the author's previous works and the unknown writings. Since the first document was created, the need to reveal the author of an arbitrary document based on the analysis of writing style has emerged as one of the most active research topics, with many potential applications in forensics. Writers tend to leave a unique fingerprint [24] in their writings unconsciously and this kind of fingerprint is called the style [23].

Looking back to history, the first attempt of writing style identification was in 1877 by Thomas Corwin Mendenhall (an American physicist and meteorologist), when he studied the word-length frequencies of writers in order to find a mathematical method for authorship attribution [34]. This style quantification approach was applied to analyze the famous "Shakespeare authorship question" (the argument that other writers, including Sir Francis Bacon and Christopher Marlowe, are the real authors of some of Shakespeare's works). [35]. In 1964, Mosteller and Wallace initiated the idea 
of applying computational and statistical fundamentals to stylometry when they used function words and Bayesian inference to find out the exact authors of the Federalist papers between James Madison and Alexander Hamilton [36]. Their creative work was the pionecr of the authorship research that quantifies writing style by using defined features, which is now known as Stylometry [51]. Stylometry is generally defined as the study of linguistic style by applying statistical analysis on the literature piece of work. Stylometry works on the assumption that the usage of words to form syntactic structures is both habitual and variable, and during this procedure, authors create individualized and unconscious patterns that can distinguish one from another [11]. Therefore, by focusing on those individually unique linguistic features with the proper classification method, we can seek to identify the author of a written work such as a document. Stylometry is not only applice to natural written language, but also to other mediums that can reflect the author's style such as computer code [9], music [48] and fine-art paintings [47]. The most common application of stylometry is for determining the authorship of unknown writings. Stylometry can be used for other purposes besides authorship attribution including multiple authorship testing [21], authenticity verification (e.g., of racial discrimination email, suicide note, relationship affair diary, etc.) [12], text genre detection [43], forensic linguistics [46], and author profiling [29].

The large growth of online communication in recent years has created the need for better analysis of wcb contents. In addition, the crimes can exploit the anonymous nature of various Internet resources such as emails, websites, and forums for communication and discussion about criminal activities. Therefore, web-based authorship identification problem is becoming increasingly important and attracting a lot of researchers.

\subsection{Dark Web forums}

Traditional Web search engines, including the powerful ones, such as Google and Bing, are unable to index everything on the Internet. In general, we can divide the 
Internet into two main parts: the visible layer (Surface Web) and the invisible layer (Deep Web). The Surface Web contains all searchable contents that are visible to the general public. In contrast to the Surface Web, the Deep Web is the section of the Internet whose contents have not been indexcd by standard scarch engines. The contents that reside on Deep Web is massive compared to the one on Surface Web. According to some studies, the data volume in Deep Web is 500 times greater than in Surface Web. Surprisingly, most people browse or visit Deep Web everyday. It can be banking accounts, Facebook conversations or Gmail inboxes. Such kinds of information will not be found on search engines as the service providers want to protect users' privacy and security. The Dark Web is a small part of the Deep Web that has been intentionally hidden and is inaccessible through common web browsers such as Safari, Chrome, Opera, Firefox ctc. It allows the users to browse and surf the Dark Web contents anonymously and secretly. Figure 2.1 helps to illustrate different layouts of the Internet.

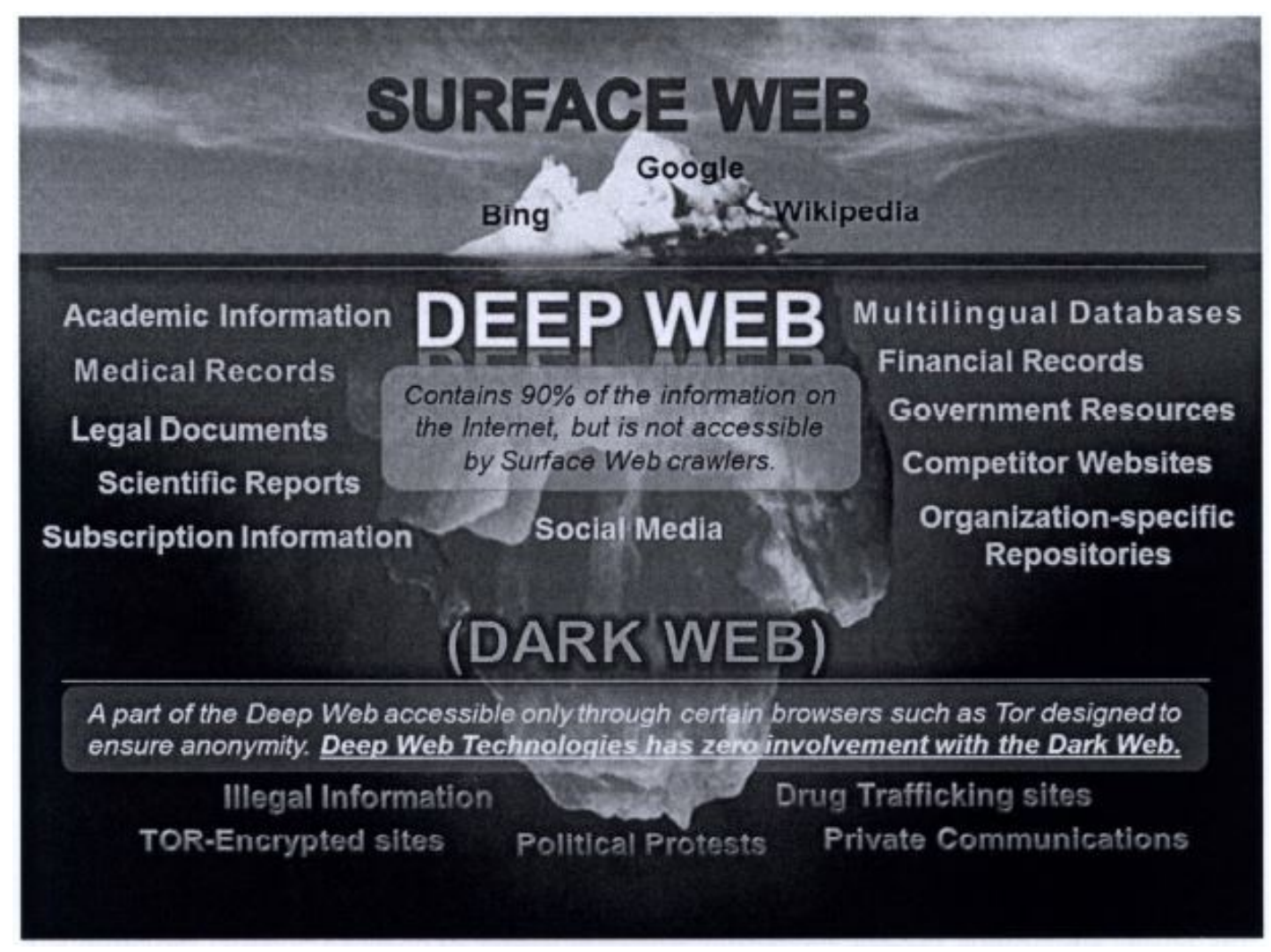

Figure 2.1: The Internet layout (from http://www.deepwebtech.com) 
The rapid growth of the Internet has given rise to the Dark Web, the problematic part of the Internet associated with cybercrimes. The most popular content that resides on the Dark Web is found in an anonymity network called TOR. In order to access TOR network, we necd to use TOR browser, a dedicated cross-platform web browser for browsing Dark Web content. TOR helps people to improve the security and privacy on the Internet by moving their traffic across virtual tumels in the TOR network, which is a collection of volunteer-operated servers.

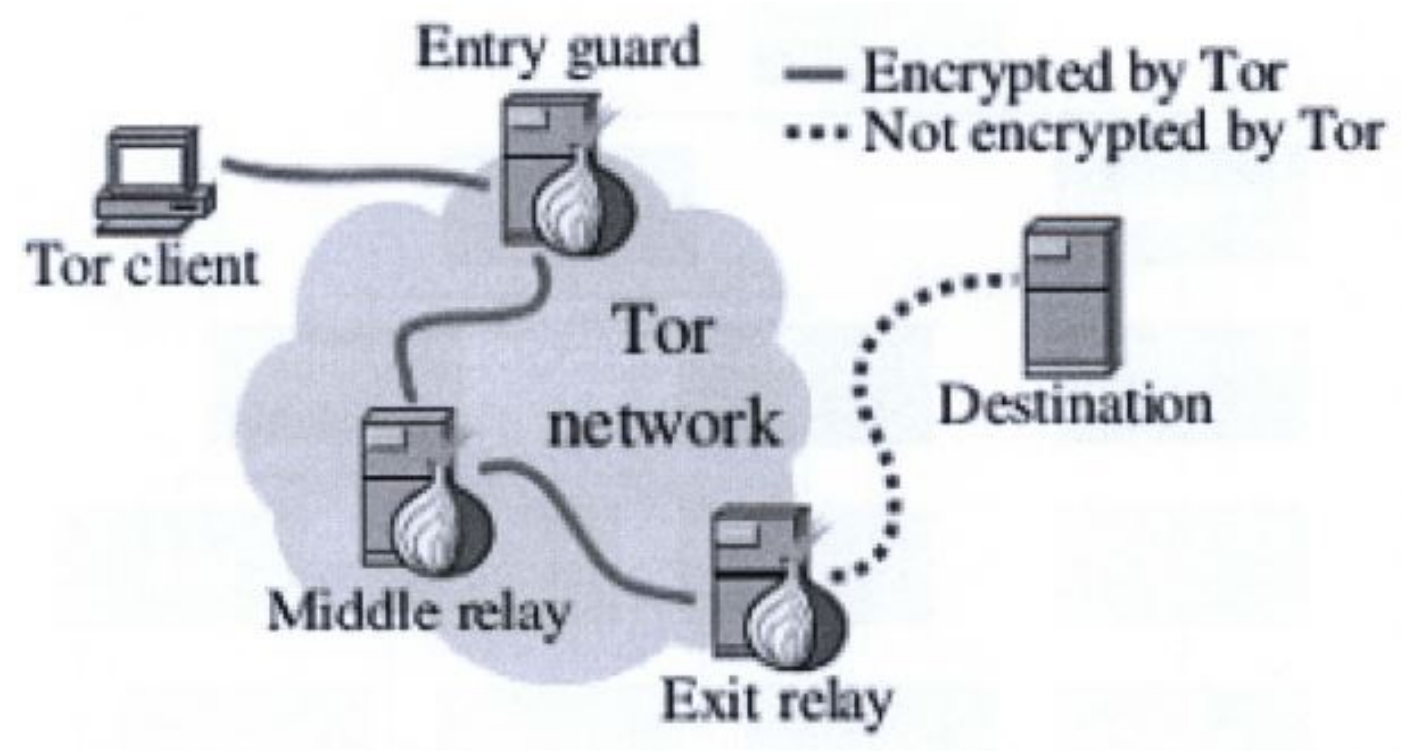

Figure 2.2: TOR's workflow (from http://arstechnica.com)

In addition, TOR is an effective tool which allows the users to bypass the Internet filtering and gain access to otherwise blocked/censored materials. A novel use of TOR is the establishment of a private communication channel which serves as a component in a bigger system. However, misuse of TOR for criminal purposes makes it a good environment, in terms of safety and privacy, for illegal activities and transactions. For example, A notable of this usage of TOR is Dark Web forums, which stays under the radar of search engines and provides a secure discussion platform for users. In particular, Dark Web forums are discussion sites supporting online conversations in the form of postcd messages. The forums are organized in a trec-like hicrarchy structure. There are multiple categories, each of which contains numerous nested subforums. Each subform contains multiple threads. User's posts of each conversation are captured in a 
thread. The thread is started with an initial post by the thread owner (creator) and the following posts are referred to as replies. The information of cach message is also recorded (poster, post timestamp, edit timestamp, etc.). Just like ordinary forums, the analysis of separate posts on forums can lead to insights regarding interactions between users' and topical trends. Figure 2.3 illustrates a typical Dark Web forum structure

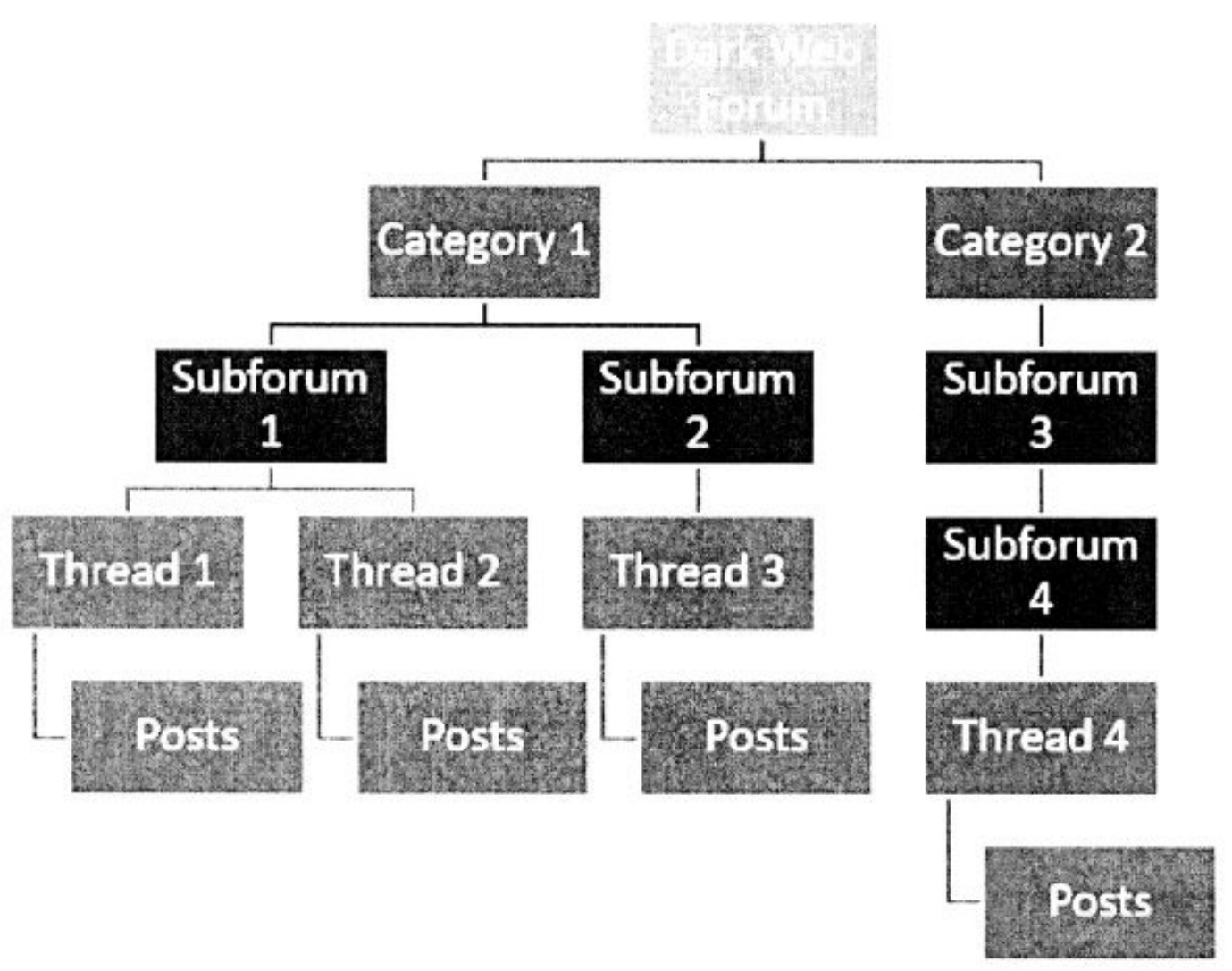

Figure 2.3: A typical Dark Web forum structure

Dark Web forums are utilized by cybercriminals to set up secret trading networks and support the exchange of illegal goods (e.g. drugs, weapons, child pornography contents etc.) and services (e.g. credit card stcalth, online credential theft, assassination, website hacking etc.). Criminals use Dark Web forums' screen names or aliases to communicate with others. In addition, the user's private identification information is usually not shown on those forums and there are no restrictions on the number of accounts a single user can create. In other words, a user can create several anonymous 
accounts on the same forum and this kind of feature creates multiple challenges to the security and intelligence organizations in tracing back the identity of the anonymous users. For example, on 3 November 2014, the then 18 years old Newcastle man Liam Lyburd was arrested because of his threat to carry out a mass-killing at Newcastle College on a Dark Web forum called "Evolution". The police discovered that he had used two different accounts with unique usernames ("The Joker" and "I Love My Anger") to express his admiration for Anders Breivik, who was sentenced 21 years in prison for killing 77 people in Norway, and Jaylen Fryberg, who carricd out a college attack in the US in which four students were killed [15].

The Dark Web and its danger to the society have extensively raised public awareness in recent years. A great number of parties, including the government and academic researchers, have paid serious attentions to this online threat. Although it is often difficult to detect the identities of Dark Web participants due to secure design of the platform, law enforcement agencies can effective identify and track illegal activities discussed in the forum. Accordingly, many researches have been carried out to study the Dark Web forum data. For example, Abbasi et al. [1] studied discussions (both English and Arabic) on Dark Web forums aiming to classify the sentiment of the opinions expressed in the discussions. Zhang et al. [56] created a Dark Web Forums Portal to collect and analyze data from multiple international Jihadist forums. We believe that the cross-association of information gathered from different forums would be effective in improving detection accuracy. This can help to counter the anonymity abusers in Dark Web forum environments. This is one of the focus of our work.

\subsection{Authorship attribution in online messaging}

One of the most important authorship attribution challenges is the identification of people involved in online conversations [13] [41]. This task has been becoming more and more essential due to the emergence of online social networks which allow users to interact in an anonymous manner via username of choice. There are several issues associated with online authorship identification compared to the traditional forms 
of writing. Message length is a major problem, especially in the case of very short messages (e.g. online forum quick chat/reply) as they make identification nearly impossible. In addition, the broad range of potential authors in online environment adds more challenges to the problem. For instance, according to a recent statistics, there are around $23 \dot{3}$ million unique users on Reddit, a very famous discussion website. Furthermore, we also need to take into account the casual nature that online users post their messages. Online messages are obviously less formal than traditional writings in the aspects that they contain improper usage of grammars, carclessly chosen structures, overuse of abbreviations and many typo errors etc. Those characteristics of online messages are usually referred as noises which make the online authorship identification task more difficult. However, not all of the traits are useless. Various identity discriminators can only be found in online messages such as greetings, signatures, quotes, links. Some users may even carclessly provide contact information such as phone number, address, email etc. in their communications. This set of features is very helpful for online authorship identification.

The most foundational work to apply authorship identification methods to the Internet environment was conducted by $\mathrm{De} \mathrm{Vcl}$ and his companies when they carried out multiple experiments on authorship identification of emails [16] [17]. Multilingual dimension was also added by other researches. For example, Zheng et al. studied multilingual web forum messages [58], mostly in English and Chinese by building an authorship analysis framework. There are two main components in that framework: feature selection which extracted style markers, structural features, and content-specific features and inductive learning techniques such as decision tree [40], backpropagation neural networks [31], and support vector machines [14]. In another direction, Ahmed et al. considered about the application of authorship analysis to Arabic web resources [1]. They dealt with different challenges of the morphological Arabic language including inflection, word length, and elongation.

Existing works for authorship identification mainly focus on three aspects: writing style features, attribution techniques, and writing languages. As we only care about 
English related messages, we are going to review the former two aspects in the following sections. We also include a short description of the relevant datasets available in the literature.

\subsection{Writing-Style Features}

It is often crucial for authorship identification to be applicable in different applications, content agnostic features are necessary. In early works, sentence length [55] and rocabulary richness [54] were proposed. These measures are based on the fact that some writers prefer simple, short sentences while others have a tendency to use complex, long sentences. Later, in 1987, Burrows created a list of common high frequency words (typically 30 or 50 words) which can be used in stylistic attribution. In addition, short words that contain two or three characters (i.e. eat, cab, in etc.) are considered by Holmes (1998). Furthermore, Holmes and Forsyth illustrated a way to select the most appropriate set of words for these word-based and character-based features [22].

The appearance frequency of function words such as "when", "while", "without" etc. in the document was initially utilized by Mosteller and Wallace in 1964 [37]. Subsequently, function words are proven to have good discriminating capability by many rescarches including Burrows (1989) Holmes (1995) and Baayen et al (1996). Due to the high frequency of function words and highly grammatical roles in the language, people seem to use them in their writing in an unconscious manner. Therefore, function words can be utilized for profiling writer's unique writing style. Other syntactic features such as part-of-specch [44] and punctuations [6] also play a crucial role in the authorship analysis processes. These studies showed that syntactic components can provide more reliable authorship identification results compared to lexical components.

In addition, proper usages of keyword-based features can improve the performance of author identification significantly. In 2003, Zheng et al. showed that the addition of some content-specific features in the context of cybercrime was useful in improving the author-identification accuracy [58]. 
Based on our observations, we can extract additional informative features from online messages that most traditional stylometry researches on written documents did not need to take care of. For example, HTML-markup related features (fonts, color, size ctc.) can be considered. Most dedicated uscrs put their efforts into making their posts attractive and unique in order to gain attention from others by creating their own style marker. In addition, non-dictionary words (slangs, emoticons, abbreviations etc.) are also being intensively used in daily online conversations which can help to reveal the users' personality as well as writing style habit. Therefore, the capture of such features can help to add more distinguishing power to our framework.

\subsection{Attribution Techniques}

Amongst the authorship attribution techniques, statistical and machine learning methods are the two most frequently used ones. Among different statistical methods, principle component analysis is the most notable and has been used to achieve high degree of accuracy [8]. The basic idea of this technique is to project the high dimensional data into the few strongest dimensions. These dimensions allow the visualization of the data on lower dimension spaces (2D or 3D) for effective understanding by analysts. The better the separation between texts written by different authors, the higher degree of accuracy that new texts can be attributed to the correct author. However, there are some complications in these approaches, including the need for more precise models and assumptions.

The exponential growth in computer power over the past several years has promoted machine learning as a better candidate to solve the problem. Machine learning consists of a variety of different techniques such as Bayesian, decision tree, neural networks, k-nearest neighbors (k-NN), support vector machines (SVMs), ctc. Wang et al. [50] pointed out two huge advantages of machine learning compared to statistical methods. Firstly, machine learning techniques are more scalable as they can handle a larger number of features. Secondly, an acceptable tolerant degree to noise can be achieved with machine learning techniques. This advantage of SVM is essential in big 
data applications which require online predictions over a high volume of data at high speed.

Among machine learning techniques, SVM classification is the most popular in recent years due to its classification power and robustness. Specifically, previous works [57] show that"SVM outperforms other classification methods such as random forest and multilayer neural network in identifying the actual author of a piece of writing. Diederich et al. first introduced SVM to this field in 2000 [18] where experiments are based on newspaper articles. One year later, they also used SVM to classify email messages belonged to 150 authors [8]. After this work, many other researchers also used SVM in their works, and the performance of SVM is further proofed [5] [45]. SVM was first introduced to the literature of machine learning by Vapnik [49]. The basic idea of SVM is to find a set of supporting examples which define the decision line which best scparates two classes of points. SVM is costly to train but very quick in operations as only the supporting examples are relevant in making predictions. Figure 2.4 is an illustration of the working of SVM. In this example, the objects belong either to class 1 (red circles) or class 2 (blue triangles). The separating line defines a boundary on the left side of which all objects bclong to class 1 and to the right of which all objects belong to class 2 . The Gap is the margin between the two parallel hyper-planes of class 1 and class 2 which we need to maximize. When working with SVMI, the principle challenge is to choose the most suitable kernel for representing the data in the feature space and compute the hyperplanes separation in order to minimize the empirical risk of wrong classifications. 


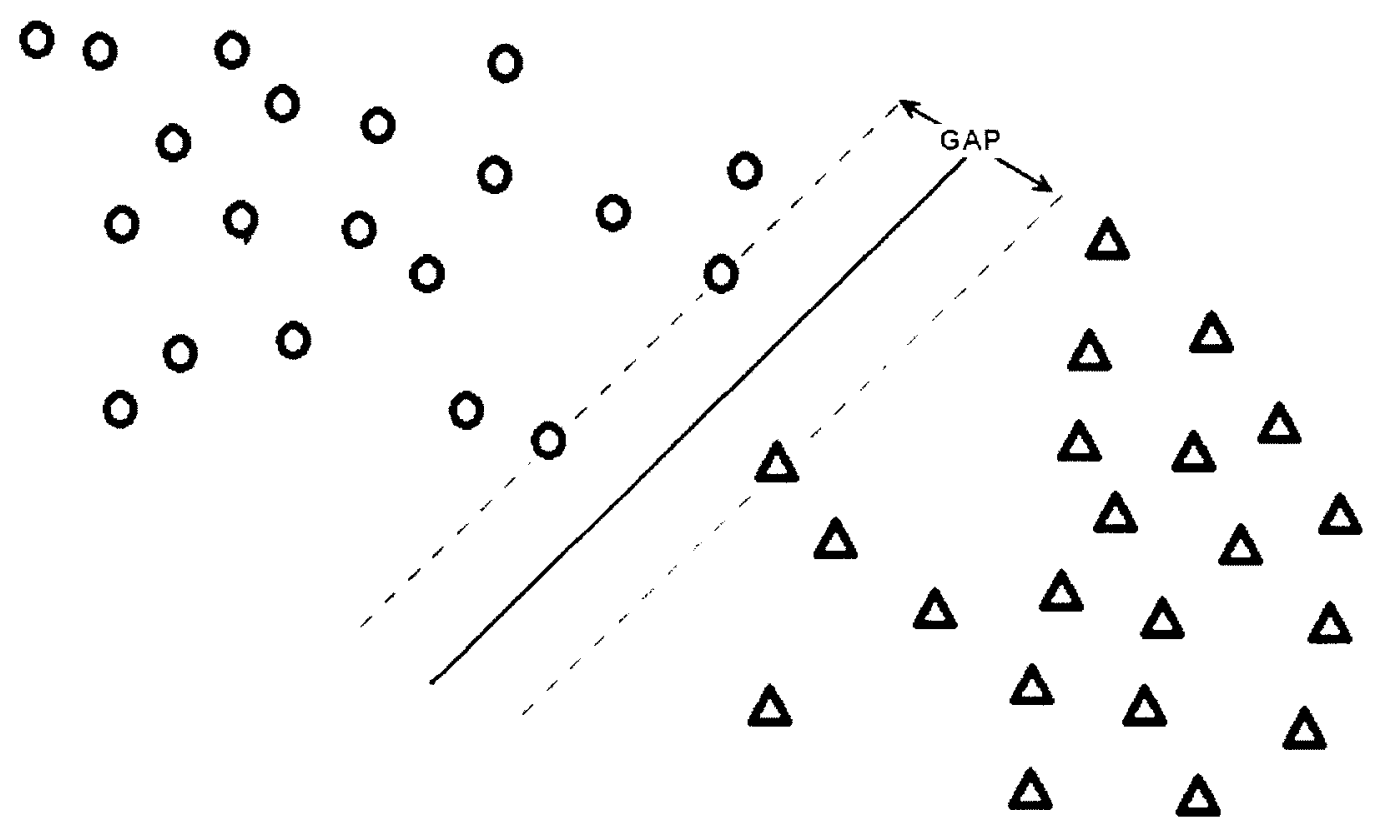

Figure 2.4: Support Vector Machines (SVM) classification

When evaluating the performance of SVM, most of the existing rescarches were evaluated by generating artificial multiple profiles per author by dividing the collected texts from an individual author into multiple separate parts [4] [30]. Based on our cxperiments, we observed that profiling authors based on texts of the same topic is easier than texts of various different topics. As a result, we have to tweak the text splitting process so that the topic distribution mentioned in each part is nearly balanced. We implemented it in an iterative manner bascd on discussed thread as each thread usually discusses about a single topic. Assume we need to create three artificial identities for a specific author. We put the first post of each user in a thread into the first part, the second post into the second part, the third post into the third part, the fourth post into the first part, and so on.

\subsection{Relevant Datasets}

There are many challenges associated with the data collection on Dark Web forums compared to surface web forums. Accessibility is a big issue as most Dark Web forums 
restrict their contents to the registered users. They are known as password-protected Web resources that require registration and login. In addition, some Dark Web forums provide the contents in the unlinked form. "Unlinked content" referred to the online pages which are not linked to by other pages. This helps to limit the capabilitics of web crawling programs. Hence, creating an effective crawling tool for Dark Web forums is a time-consuming and uneasy task. For the purpose of this research, we use an existing data source that is available online. The bulk of our data was obtained from a vast archive compiled by Gwern Branwen, who is an independent Dark Net Market (DNM) researcher. Dark Net Markets (DNMIs) are online markets which are typically hosted as TOR hidden services. They provide a platform in which buyers and sellers can do business using digital currency (the most famous one is Bitcoin [38]).

The most famous DNM was "Silk Road" which has generated about $\$ 1.2$ billion in revenue since its creation in 2011. "Silk Road" was created by Ross Ulbritch who lives in Texas and holds a master's degree in materials science and engineering at Pennsylvania State University. In October 2013 the Federal Bureau of Investigation (FBI) took legal actions on "Silk Road" by shutting down the site and arresting its founder (Ross Ulbritch). After that, on 6 November 2013, some former administrators of "Silk Road" created the next generation of "Silk Road" which is known as "Silk Road 2.0". "Silk Road 2.0" and its former "Silk Road" are best known as online platforms for selling and discussing about illegal drugs.

The full archive is in the Torrent format. There are around 89 markets, more than 37 forums and 5 other sites in the archive, which represent more than 4000 mirrors of more than 40 millions of files. The approximate size of the data in compressed format is around 49.4 GB (163 compressed files in total), which can be unpacked to more than 1548 GB. This dataset mainly contains HTML, CSS, and images. The archived data can be uncompressed on both Mac and Windows operating system (OS) using either the built-in OS archiver or external programs such as 7zip, Stuffit, or WinRAR. Most of the material in the archive dates from 2013 to 2015. 


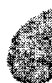

\section{Research Problems and Methods}

In this section, we discuss the scenario where our research would apply on, the motivation behind our experimental study and what we want to achieve from the experiments. In addition, we also introduce our proposed framework that can be useful for the authorship matching of Dark Web online messages.

\subsection{User Identity Matching across Dark Web}

In addition to traditional authorship identification problems, we define the authorship matching problem as the task to estimate the writing style similarity between multiple users (having the same username) on multiple Dark Web forums. The problem can become very complex if the users purposely alter and obfuscate their writing style in order to make sure that they are completely anonymous. Kacmarcik et al. [25] have explored different techniques of automatically obfuscating a document to preserve anonymity and found that the number of changes per 1000 words is too small to confound the standard approaches to authorship attribution. For this research, we assume that the authors do not attempt to hide their writing style when participating in online discussions on Dark Web forums. The ultimate purpose of this task is to figure out whether two accounts having the same username on multiple Dark Web 
forums belong to the same person or not. The chance that two different users coincidentally create accounts with the same username on two different forums is not rare. For example, two users who are the fans of Marvel comics/movies may create the accounts with the same uscrname "spiderman". This document proposes a novel approach for performing authorship attribution matching using stylometry and basic classification technique (SVM).

\subsection{Research Objective}

As seen from our study in the previous sections, we find that suspects of many real world online criminal investigation cases can be narrowed down if authorship matching of Dark Web users is adequately addressed. In this Master candidature, our objective is to study the problem of authorship matching of Dark Web users with same username on multiplc Dark Web forums. As with standard classification, we focus on both theoretical and practical aspects of this problem. In particular, the research aims to address the following issues:

(i) We introduce a new novel framework to tackle the problem of authorship matching with the objective to utilize the current state-of-the-art methods of authorship attribution for effectively tracing users' identities on Dark Web.

(ii) We carry out a series of experiments to evaluate our authorship matching algorithms on the Dark Web archived data corpus. The insights, revealed through the experiments, are invaluable feedbacks to improve the performance of our framework and lead to a better understanding of the authorship matching problem.

\subsection{Framework}

Basically, authorship identification process can be divided into five steps according to Figure 3.1. In the first step, we extract forum messages from the Dark Web archived data. The raw data is then passed to the Data Filter layer which helps to take 
out information that is useless or information that can be confusing. The third step is to extract useful information (features) for stylometry which is represented in the vector format. Feature extraction is the preliminary step to prepare the dataset before dividing it into the training set and the testing set. The weights of the classification model are adjusted based on the performance on the training set (step 4). Finally, the generated model is used to test against the testing set to generate the matching results (step 5). The details of each step are described in the subsections below.

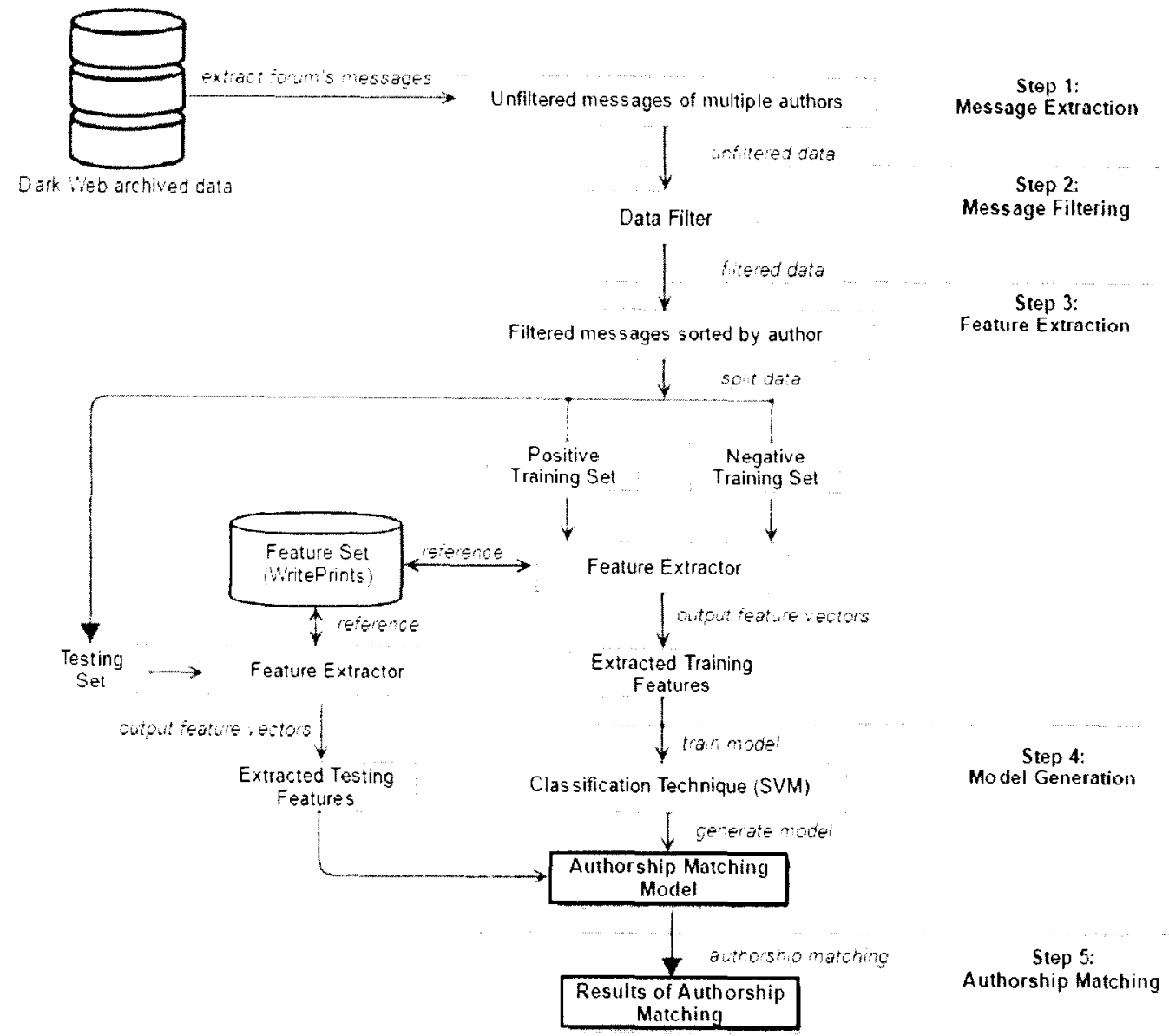

Figure 3.1: Authorship matching process pipeline

\subsubsection{Message Collection}

In the first stcp, we necd to define the list of authors that we want to analyze and extract a set of forum messages posted by those authors from the Dark Web archived 
data. Figure 3.2 is an example to illustrate the structure of user's posts in Dark Web forums.

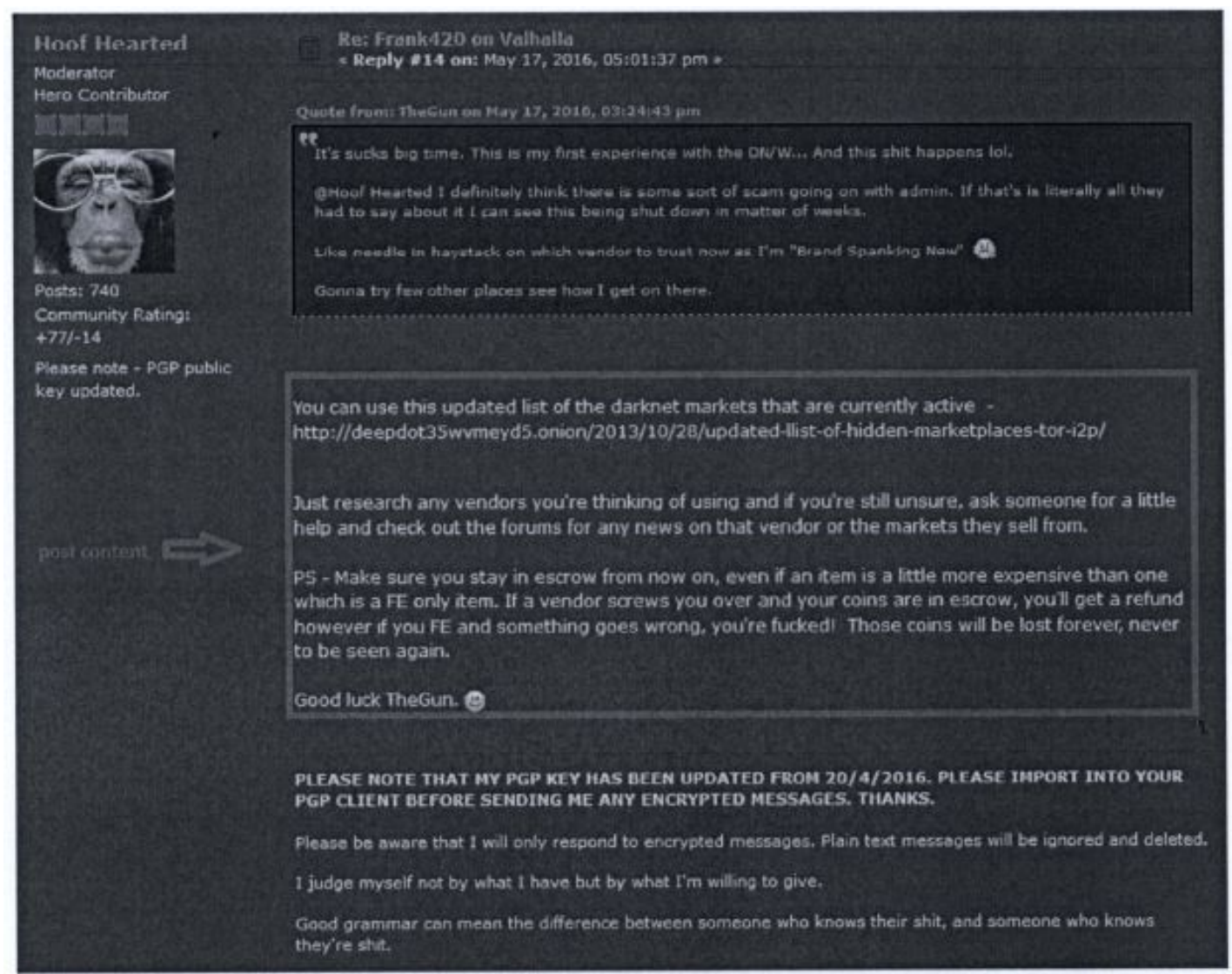

Figure 3.2: A sample user's post in a Dark Web forum

As the archived data is in HTML format, a data transformation step is required to convert the HTML documents into raw plain text documents. In each post block, there are many redundant components that we need to remove such as user's profile details, user's signature and quoted post of another user etc. The preprocessor first parses an HTML document and returns the actual user's post contents. The parsing process is done with the help of "BeautifulSoup", a python library for HTML parsing. Basically, "BeautifulSoup" helps to extract the raw text data from the HTML contents through the removal of unnecessary markups and tags. We first need to inspect the HTML contents and tell "BeautifulSoup" where it needs to traverse to and extract the text data (by spccifying the identities of the desired HTML elements). Figure 3.3 
shows a sample parser process which transforms the original complex, multiple nested HTML hierarchy structure into plain text document format.
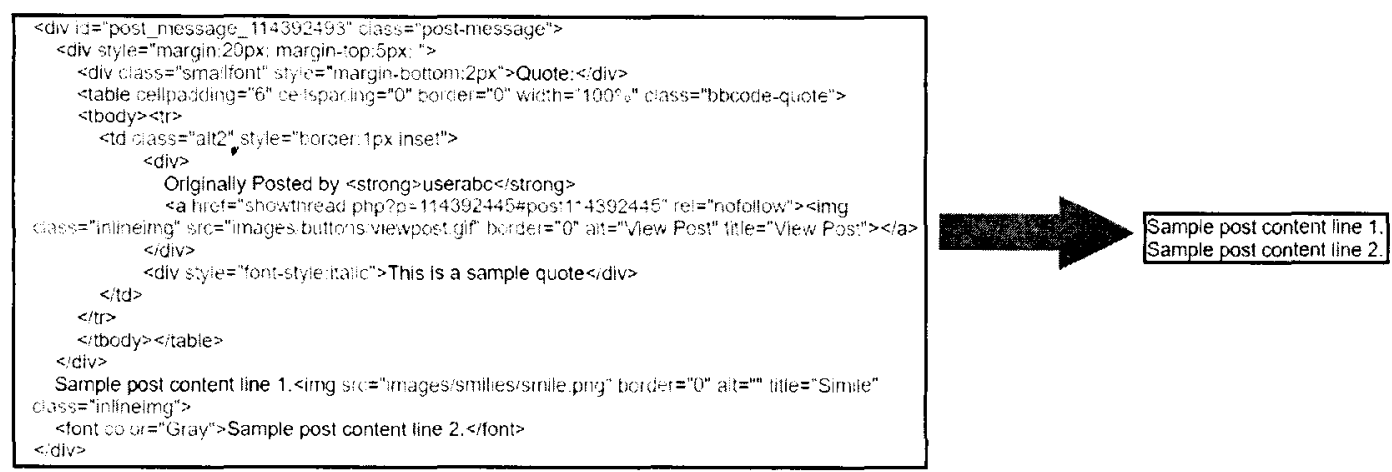

Figure 3.3: HTNL to raw text data transformation using parser

After the message collection process finished, we organized the messages into individual author's document. Each author's document should contain at least 6000 words of online messages (forum posts). The document is formed by combining multiple individual users forum posts consecutively, separated by a blank line. A sample of an author's document that contains multiple forum posts is captured in Figure 3.4.

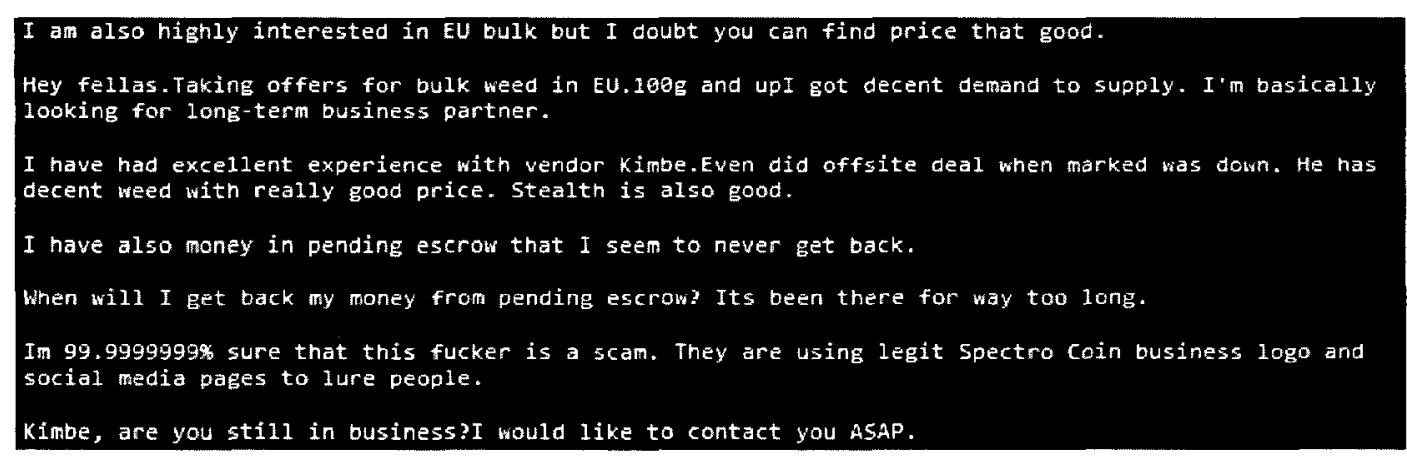

Figure 3.4: A sample part of an author's document

\subsubsection{Message Filtering}

Real-world classification problems are influenced by several components, which may negatively impact the accuracy. The most important factor is noise. The performance of the authorship matching model built under noisy circumstances does not only depend on the quality of the training data, but also on its robustness against the noisy data. The noise in text data is defined as the undesired blocks of words which 
provide no or very little information and need to be removed in order to enhance the total quality of the classification process.

For Dark Web online messages between users, one of the most common "noise" which can be casily observed is known as users' PGP key. PGP stands for Pretty Good Privacy. PGP is most commonly used for data encryption and digital signatures. It adopts the public-key encryption mechanism in which a public key is used to encrypt the data and another separate private key is used to decrypt the encrypted data. Dark Web users usually make use of PGP to encrypt their sensitive information when doing illegal trading. Merchants on underground marketplaces usually provide own public key on their profile. Whenever other users want to trade goods or services from a merchant, they need to encrypt their shipping address using the public key provided by that morchant. In this way, only the merchant can decrypt and view the buyers' messages.

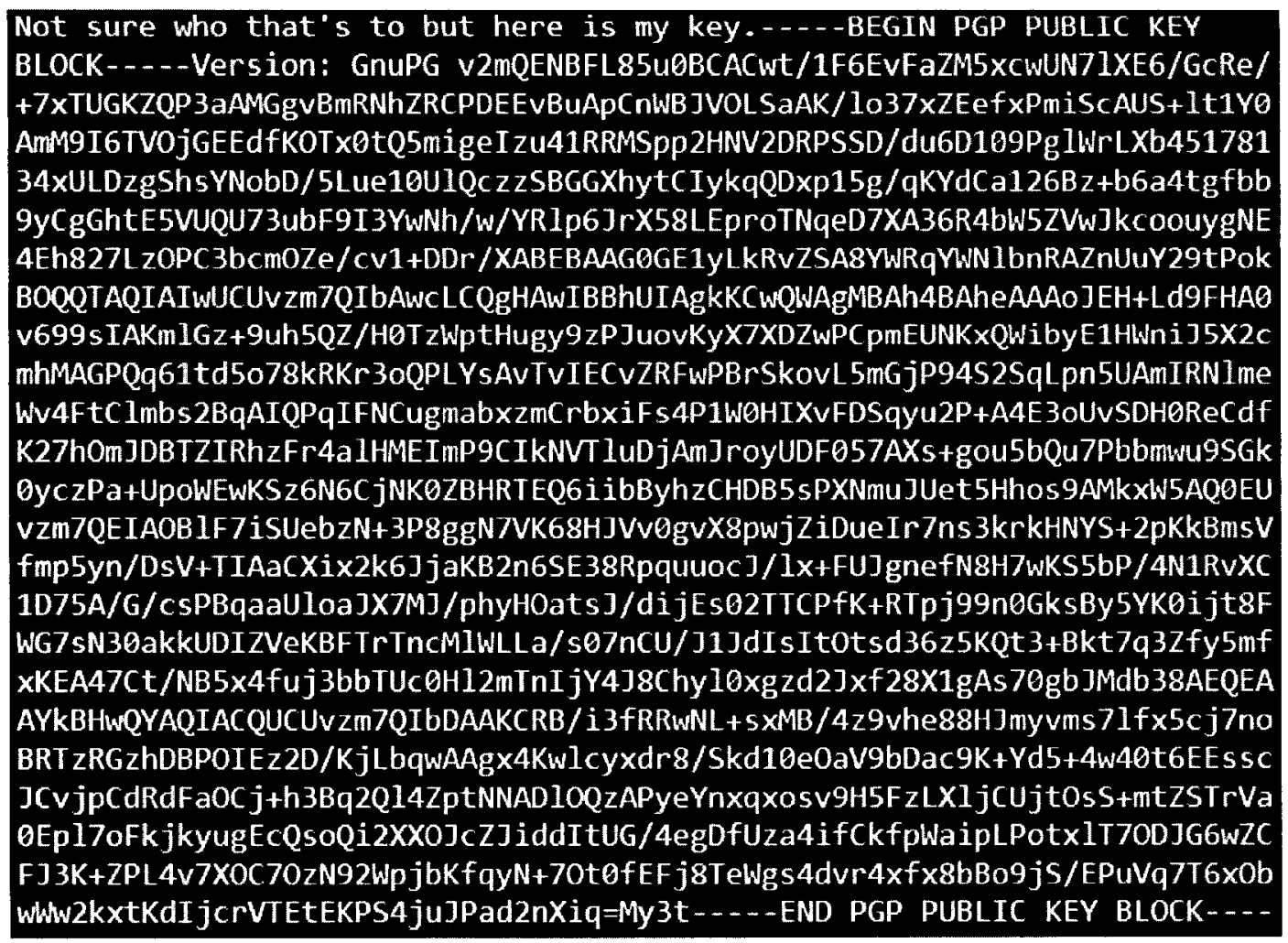

Figure 3.5: A sample conversation including PGP encryption's texts

Another common "noise" that we need to consider comes from the hyperlinks 
(urls) that appear in the user's posts. Almost no user can remember a long and difficult to remember url and retype it. In fact, users tend to copy/paste the url when they want to refer to an external source in their posts. Although each hyperlink is not directly typed by the authors, it still generates features for the stylometry process. For example, most of the feature set used in stylometry include bigrams and trigrams. The hyperlink usually begins with "http" or "https" and thus will create bigrams ("ht", "tt", "tp") and trigrams ("htt", "ttp"). These features contribute little to the stylometry process and may even affect the matching results badly if they dominate other features. Therefore, noise removal is a necessary step to remove such kinds of nonsense data and improve the total quality of the classification process.

In addition, as our research mainly focuses on English text data, we need to remove the chunk of texts that are written in other languages. Language detection is performed using a Python module called "guess_language", which adopts a trigram-bascd algorithm. "guess_language" can detect over 60 languages, including non-alphabetical ones such as Japanese, Chinese, Korean, and Greek. The module receives lines of texts as input and returns the predicted language. The result is in the form of language abbreriation ('en' stands for English, 'ja' stands for Japancse etc.).

Last but not least, we need to remove duplicated paragraphs from the text documents. For example, some vendors tend to spam advertisements about their products or some admins may post the forum rules and advices multiple times to remind the users. Another common duplication problem comes from the user's forum signature. Most forums allow the users to add their unique signature in their user profile that is automatically appended to each of their posts. As a result, it reduces the exact length of the documents that are used in the classification. All the stylometry methods require some minimum number of words for both training and testing data. The more the duplications are present, the less effective the classification process become. In order to deal with this problem, we followed the following step. We scan through all of each user's posts and search for common substrings. Those are the obvious cvidence of uscr's spams or signatures that need to be filtered out. 


\subsubsection{Feature Extraction}

After the "Message Filtering" step, we divide the data into separate training and testing dataset. As in other classification problems, there are two subsets of the training set which are positive training set and negative training set. The authorship matching problem requires some known author profiles (positive data instances) such that we need to match (label) each of the testing data to one of them. The details of different divided components of the dataset will be described in details in chapter 6 (Experiments).

The purpose of the feature extraction process is to convert the raw text data into informative and non-redundant data representation to facilitate the subsequent learning and generalization steps. The raw collected messages are in textual fragments, which are unstructured and not ready to train with machine learning algorithms. Therefore, we first transform the messages into structured form by extracting representational features which greatly affect the performance of the authorship attribution algorithm introduced previously. These features are referred hereafter as writing style features and include some special features which are not used in the authorship attribution of traditional writings. Compared with formal publications such as literary works or published articles, online messages have some unique characteristics which may help to reveal the writing style of the individual author. Because of the casual nature of the Web-based environment, authors are more likely to leave their own writeprints in their online articles (email, chat text, forum posts etc.). Many users try hard to differentiate themselves from other by posing some unique and special writing style online. For example, some authors have the habit of ending the online posts with a smile emoticon ":)" because they think it's cool. In another case, some people, especially the non-11ative English speakers, tend to repeatedly use some misspelled words in their sentences. Common examples include many daily conversational words such as: "absense", "acknowlege", "noticable", "succesful" For the Dark Web environment that we consider in this research, there are additional fingerprints that can be taken into accounts. For example, drug-addicted users may mention a lot about a 
specific drug name or an active vendor will copy/paste an advertising of his product on multiple Dark Web forums. As a result, with a carefully selected set of features that can represent those fingerprints, the performance of the authorship matching can become reasonably good.

There are two steps in the feature extraction process. The first step is to extract the features from the training dataset. After that, the next step is to extract the features from the testing dataset based on the same feature set generated in the first step. Therefore, the dimensions of both extracted training features and testing features are equal.

\subsubsection{Model Generation}

In this phase, we created predictive models based on the training features. Multiple instances of models are created. The number of generated models depends on the number of users in the positive data instances. Each model can be view as a single author's writing style profile. Each author's profile can answer whether a piece of unknown writings in the testing belong to this author or not. More details and explanations of this process are provided in chapter 6 (Experiments).

\subsubsection{Authorship Matching}

After the model generation phase completed, we can use the model to calculate the writing style similarity between the user accounts that are suspected to belong to the same person. The higher the similarity score between the two writing pieces, the more confidence we can link the profiles of the two user accounts. The result will help the interested parties to gather more information about the suspects and assist in tracing the criminal identities more effectively. 


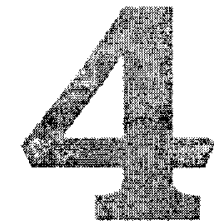 Experiments}

We conducted an experimental study to evaluate the proposed framework. In particular, we want to analyze the effects of feature types and classification techniques on authorship matching of Dark Web users. Most authorship experiments usually follow a similar machine learning pattern: the model is initially trained on a set of texts (training set) and then tested on another set of texts (test set). The data in both the training and testing set contain the feature vectors extracted from the authors' posts on Dark Web formms. The cvaluation is based on the accuracy of the experiment on the testing dataset. We apply the described train-and-test process to examine the effectiveness of our proposed authorship matching techniques by designing two different author-matching tasks.

In general, the purpose of authorship matching task is to compare each anonymous identity against all known entities (authors) through writing style classification. The identity of the anonymous author is linked to that entity with the highest similarity score. Figure 4.1 helps to illustrates the authorship matching task. 


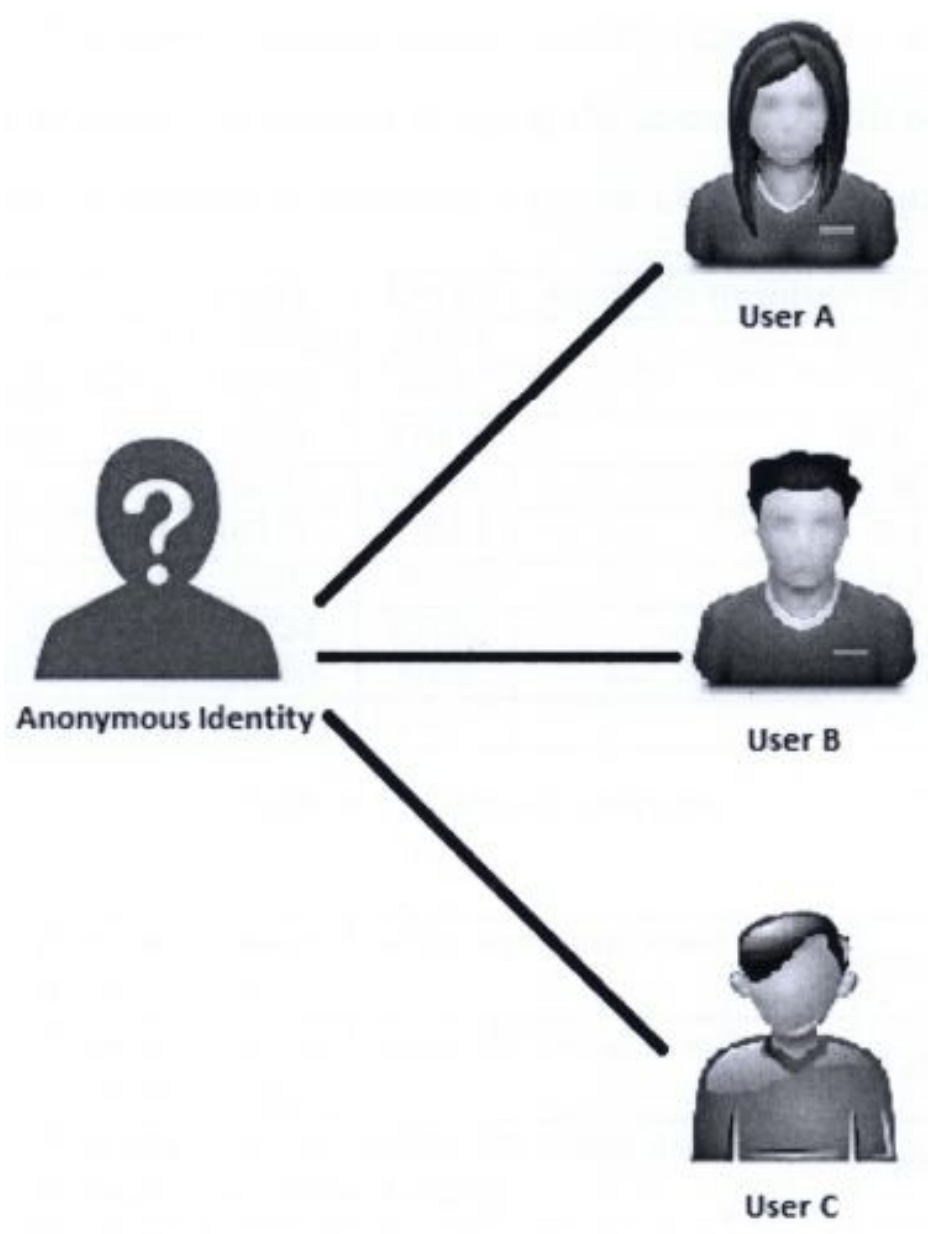

Figure 4.1: Authorship matching task

In the first experiment, we collect all the posts of a user in the same Dark Web forum and split them into two parts with nearly equal size and evaluate the authorship matching between those two parts using the proposed framework. This experiment is used as the testbed process to validate different algorithms and parameters. We label this process as "Validation Phase".

After the "Validation Phase" is completed, we proceed to the "Testing Phase". In this experiment, we identify some users that have accounts on multiple Dark Web forums. For each user, we collect his/her posts in two arbitrary forums and evaluate the authorship matching between those two parts. 
Table 4.1 and Table 4.2 provide some statistics about the dataset that we use in our experiments. The former lists the major forums in the dataset with overall details (e.g. the number of posts, the number of users, the average length of posts) while the latter summarizes the number of matching between users in multiple forums.

\begin{tabular}{|c|c|c|c|}
\hline Forum & Posts & Users & Average number of words per post \\
\hline Agora & 17248756 & 17188 & 66.296 \\
\hline Black Market Reloaded & 105732 & 8396 & 24.714 \\
\hline Dream Market & 14984 & 57010 & 29.5 \\
\hline Evolution & 514687 & 22150 & 73.736 \\
\hline Nucleus & 10345 & 104395 & 39.461 \\
\hline Pandora & 7233 & 47017 & 40.166 \\
\hline Silk Road 2 & 826734 & 22756 & 59.777 \\
\hline The Hub & 109560 & 6642 & 51.461 \\
\hline The Majestic Garden & 58293 & 3529 & 25.5 \\
\hline
\end{tabular}

Table 4.1: Dataset overview

\begin{tabular}{|l|c|}
\hline $\begin{array}{l}\text { Number of users having the same username } \\
\text { in two forums }\end{array}$ & 627 \\
\hline $\begin{array}{l}\text { Number of users having the same username } \\
\text { in three forums }\end{array}$ & 124 \\
\hline $\begin{array}{l}\text { Number of uscrs having the same username } \\
\text { in more than three forums }\end{array}$ & 22 \\
\hline $\begin{array}{l}\text { Number of users having the same username } \\
\text { in multiple forums (>= 2 forums) and satisfy } \\
\text { the post length requirement }\end{array}$ & 17 \\
\hline
\end{tabular}

Table 4.2: Dataset matching users statistics

\subsection{Validation Phase}

The main dataset used in this experiment consists of the user's posts on "Silk Road 2.0" which are extracted from the Dark Web archived data as stated earlier. We identified 10 of the most active authors with at least 400 posts and around 6000 words (the contents must be written mostly in English) who also have accounts on another Dark Web forum and cullected all of their posts. The reason for the number of words requirement originates from Eder's research [19] which shows that the sample size must be at least 5000 words in order to efficiently recognize authorial uniqueness. We denote this set as $A$. In this validation phase, we divide $A$ into two parts, each 
of which contains half the posts of each user. We denote those two subsets of $A$ as $A_{1}$ and $A_{2}$ respectively. In general, there are 10 pairs of texts in $A_{1}$ and $A_{2}$ that we need to determine the similarity. The matching result is expected to be high as each pair of text is absolutely written by the same person. This experiment serves as the validation step which can help to determine the best parameter settings for our model. We also collect posts of 99 other active users on "Silk Road 2.0" which are used as the fixed negative data instances for both experiments and denote this set as $C$.

The data in $A_{1}$ is used as the positive training data while the data in $C$ provides the negative points which help to shape the classifiers. The desired result is 10 different classifiers for 10 testing users. Specifically, the classifier of each user $U$ is constructed from the training dataset which contains posts of 100 authors (posts of 99 uscrs in set $C$ and posts of user $U$ in $A_{1}$ ). The traincd classificrs will be tested against the testing data in the set $A_{2}$ using different combinations of parameters. Multiple metrics such as Accuracy (A), Precision(P) and Recall(R) are used to assess the performance of the experinental parameters. Figure 4.2 illustrates the workflow of the validation process, in which the data in set $A_{1}$ and set $C$ form the training data which is used to create 10 different classifiers to test against the data in set $A_{2}$. 


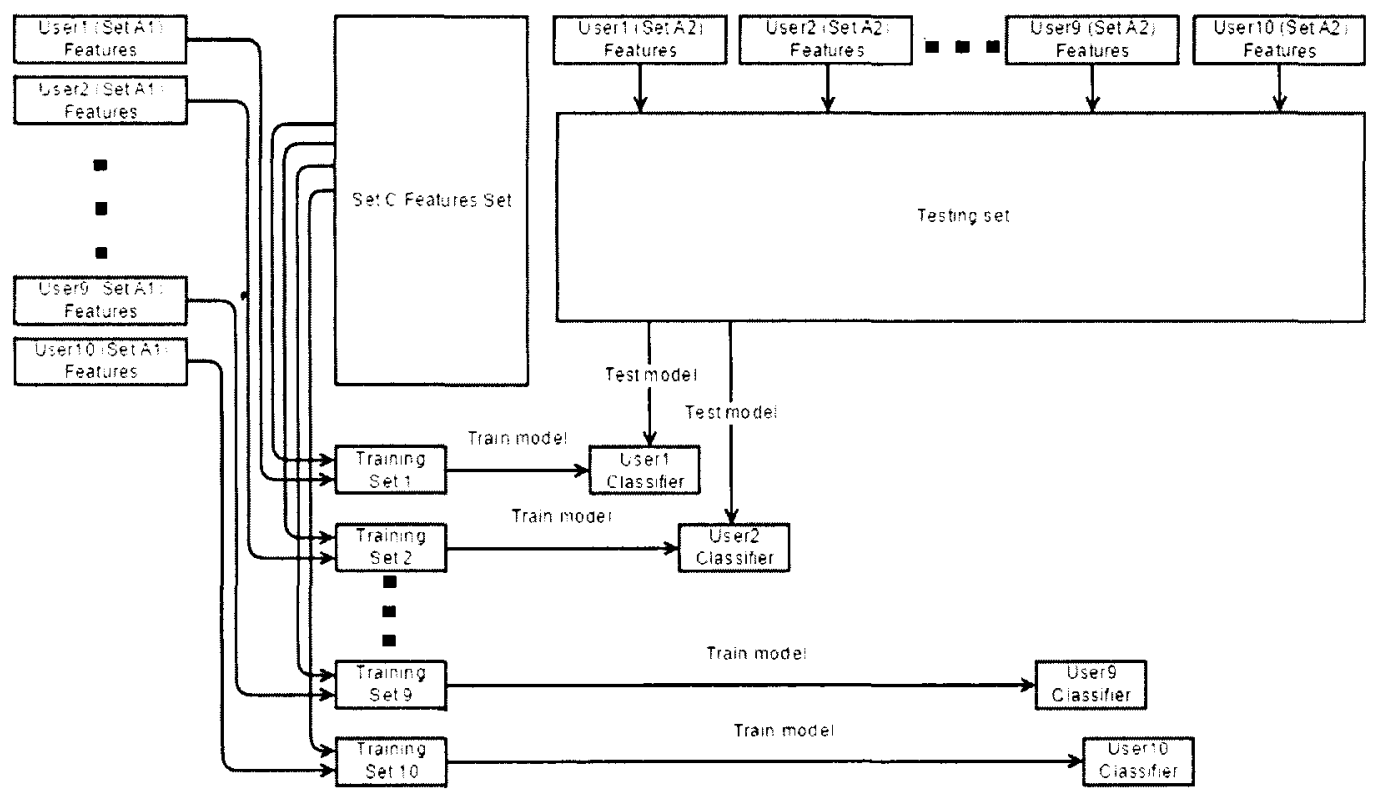

Figure 4.2: Validation experiment details

Table 4.3 summarizes the details of the data containing in training and test set in the validation experiment (set $A_{1}$ and $B$ respectively). The authors' uscrnames are abbreviated in order to protect their personal identity and privacy. 


\begin{tabular}{|c|c|c|c|c|}
\hline Type & Username/Author & Forum & Abbreviation & No.of words \\
\hline Training & $\mathrm{BM}$ & Silkroad2 & $\mathrm{BM}(\mathrm{tr})$ & 6009 \\
\hline Training & $\mathrm{CW}$ & Silkroad2 & $\mathrm{CW}(\mathrm{tr})$ & 5999 \\
\hline Training & $\mathrm{cv}$ & Silkroad2 & $\mathrm{cv}(\mathrm{tr})$ & 6023 \\
\hline Training & $\mathrm{FT}$ & Silkroad2 & $\mathrm{FT}(\mathrm{tr})$ & 6006 \\
\hline Training & $\mathrm{Ka}$ & Silkroad2 & $\mathrm{Ka}(\mathrm{tr})$ & 6014 \\
\hline Training & $\mathrm{ma}$ & Silkroad2 & $\mathrm{ma}(\mathrm{tr})$ & 5997 \\
\hline Training & $\mathrm{mu}$ & Silkroad2 & $\mathrm{mu}(\mathrm{tr})$ & 6027 \\
\hline Training & $\mathrm{ri}$ & Silkroad2 & $\mathrm{ri}(\mathrm{tr})$ & 6003 \\
\hline Training & $\mathrm{Ta}$ & Silkroad2 & $\mathrm{Ta}(\mathrm{tr})$ & 6007 \\
\hline Training & $\mathrm{Zi}$ & Silkroad2 & $\mathrm{Zi}(\mathrm{tr})$ & 6009 \\
\hline Testing & $\mathrm{BM}$ & Silkroad2 & $\mathrm{BM}(\mathrm{t})$ & 6025 \\
\hline Testing & $\mathrm{CW}$ & Silkroad2 & $\mathrm{CW}(\mathrm{t})$ & 5999 \\
\hline Testing & $\mathrm{cv}$ & Silkroad2 & $\mathrm{cv}(\mathrm{t})$ & 6001 \\
\hline Testing & $\mathrm{FT}$ & Silkroad2 & $\mathrm{FT}(\mathrm{t})$ & 5998 \\
\hline Testing & $\mathrm{Ka}$ & Silkroad2 & $\mathrm{Ka}(\mathrm{t})$ & 6012 \\
\hline Testing & $\mathrm{ma}$ & Silkroad2 & $\mathrm{ma}(\mathrm{t})$ & 6009 \\
\hline Testing & $\mathrm{mu}$ & Silkroad2 & $\mathrm{mu}(\mathrm{t})$ & 6022 \\
\hline Testing & $\mathrm{ri}$ & Silkroad2 & $\mathrm{ri}(\mathrm{t})$ & 5999 \\
\hline Testing & $\mathrm{Ta}$ & Silkroad2 & $\mathrm{Ta}(\mathrm{t})$ & 5993 \\
\hline Testing & $\mathrm{Zi}$ & Silkroad2 & $\mathrm{Zi}(\mathrm{t})$ & 6005 \\
\hline
\end{tabular}

Table 4.3: Expcriment 1 datasct overview

\subsection{Testing Phase}

In the sccond experiment, for cach uscr, we find another account in a different Dark Web forum who has the same username and collect all of his forum posts. There can be multiple accounts on multiple Dark Web forums having the same username. However, we can not anyhow select the accounts for this experiment arbitrarily. The selection of users must strictly follow the data length requirements as stated in the "Validation Phase" section (having at least 400 posts with length around 6000 words). We denote this set of data as $B$. The optimal model trained in the validation phase is applied to Set $B$. The working process of this testing phase as depicted in Figure 4.3 is similar to the validation phase, except that the data of the test set comes from set $B$ 


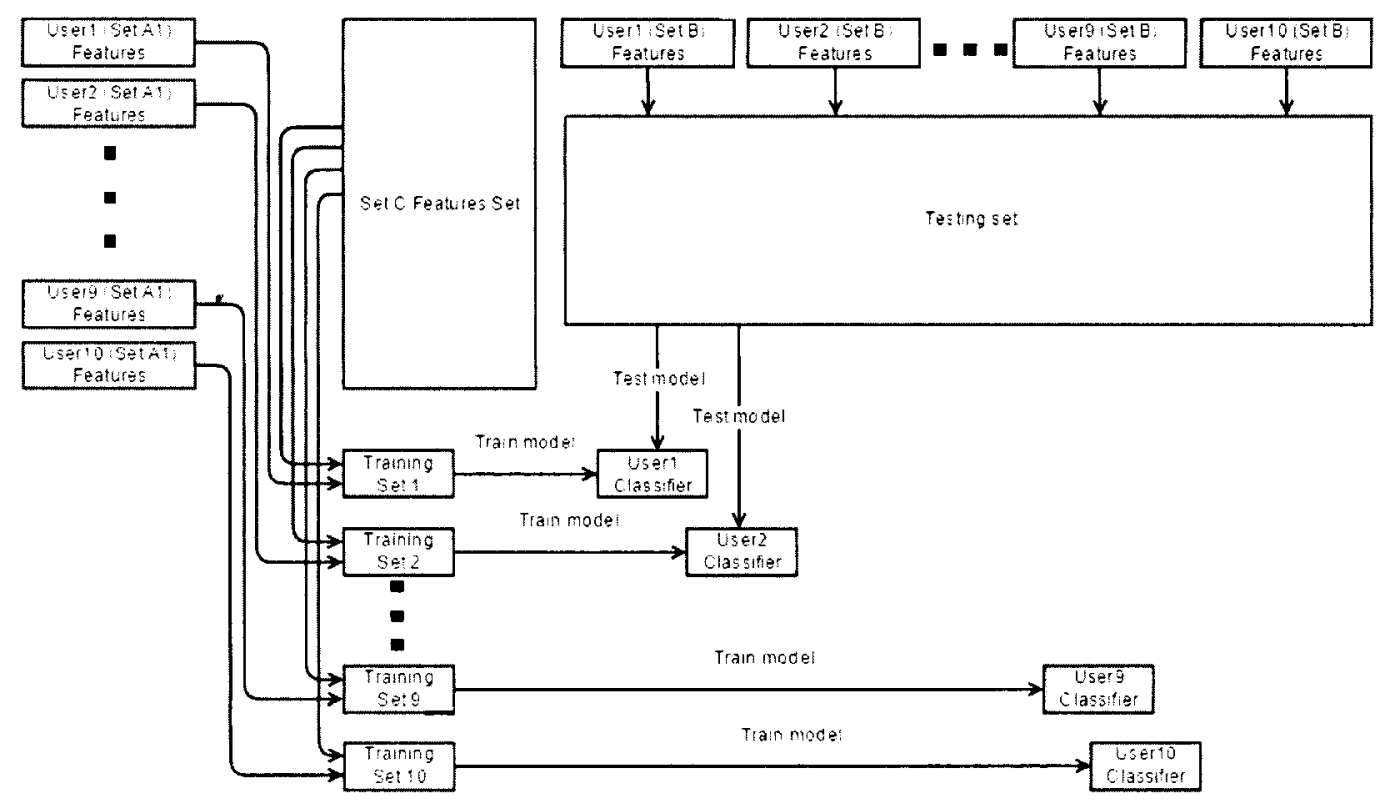

Figure 4.3: Testing experiment details

Table 4.4 summarizes the details of the data containing in training and testing sets in the testing experiment (set $A_{1}$ and $A_{2}$ respectively). We can see that the testing data is collected from various Dark Web forums such as "Evolution", "The Hub" and "Agora" besides "Silk Road 2". These are other large discussion and market portals that reside on Dark Web. "Evolution" is a large drug market which also provides wholesaling of credit card data. After "Evolution" closed in March 2015, "Agora" replaced it as the largest drug darknet market. "The Hub" focuses on darknet market reviews about different topics including drugs, crypto currency, and security. 


\begin{tabular}{|c|c|c|c|c|}
\hline Type & Username/Author & Forum & Abbreviation & No.of words \\
\hline Training & $\mathrm{BM}$ & Silkroad2 & $\mathrm{BM}(\mathrm{tr})$ & 6009 \\
\hline Training & $\mathrm{CW}$ & Silkroad2 & $\mathrm{CW}(\mathrm{tr})$ & 5999 \\
\hline Training & $\mathrm{cv}$ & Silkroad2 & $\mathrm{cv}(\mathrm{tr})$ & 6023 \\
\hline Training & $\mathrm{FT}$ & Silkroad2 & $\mathrm{FT}(\mathrm{tr})$ & 6006 \\
\hline Training & $\mathrm{Ka}$ & Silkroad2 & $\mathrm{Ka}(\mathrm{tr})$ & 6014 \\
\hline Training & $\mathrm{ma}$ & Silkroad2 & $\mathrm{ma}(\mathrm{tr})$ & 5997 \\
\hline Training & $\mathrm{mu}$ & Silkroad2 & $\mathrm{mu}(\mathrm{tr})$ & 6027 \\
\hline Training & $\mathrm{ri}$ & Silkroad2 & $\mathrm{ri}(\mathrm{tr})$ & 6003 \\
\hline Training & $\mathrm{Ta}$ & Silkroad2 & $\mathrm{Ta}(\mathrm{tr})$ & 6007 \\
\hline Training & $\mathrm{Zi}$ & Silkroad2 & $\mathrm{Zi}(\mathrm{tr})$ & 6009 \\
\hline Testing & $\mathrm{BM}$ & Evolution & $\mathrm{BM}(\mathrm{t})$ & 6005 \\
\hline Testing & $\mathrm{CW}$ & The Hub & $\mathrm{CW}(\mathrm{t})$ & 6023 \\
\hline Testing & $\mathrm{cv}$ & Evolution & $\mathrm{cv}(\mathrm{t})$ & 5981 \\
\hline Testing & $\mathrm{FT}$ & The Hub & $\mathrm{FT}(\mathrm{t})$ & 6019 \\
\hline Testing & $\mathrm{Ka}$ & Agora & $\mathrm{Ka}(\mathrm{t})$ & 6027 \\
\hline Testing & $\mathrm{ma}$ & The Hub & $\mathrm{ma}(\mathrm{t})$ & 5999 \\
\hline Testing & $\mathrm{mu}$ & Agora & $\mathrm{mu}(\mathrm{t})$ & 6016 \\
\hline Testing & $\mathrm{ri}$ & Evolution & $\mathrm{ri}(\mathrm{t})$ & 6008 \\
\hline Testing & $\mathrm{Ta}$ & The Hub & $\mathrm{Ta}(\mathrm{t})$ & 5996 \\
\hline Testing & $\mathrm{Zi}$ & The Hub & $\mathrm{Zi}(\mathrm{t})$ & 6013 \\
\hline
\end{tabular}

Table 4.4: Experiment 2 dataset overvicw

\subsection{Feature Selection}

As mentioned in the Introduction part, among the techniques that could be used to attribute authorship to anonymous or disputed documents, we focused on stylometry. The most common technique to stylometry includes two steps. We divide the set of documents into two subsets: the set of documents with known authors (training set) and the set of documents with unknown authors (test set). The first step is to gencrate a training classificr from the training set. After that, the second step is to utilize the created classifier to determine the authorship of every document in the test set. In this whole process, the main factor that affects the stylometry result is the set of features used. The number of features can become extremely large. For example, English letter n-grams consist of $26^{n}$ fcatures. Therefore, it is necessary to carcfully select the vital features that best represent the authorship uniqueness.

We have decided to implement the feature extraction process via JStylo [33] (which has been released under the free General Public License (GPL 3) and can be used 
for study and research stylometry problems). The feature extraction process helps to transform the raw text format post data into high-dimensional feature vectors which are the input to our classifiers.

In addition, for simplification and higher performance, another heuristic is adopted for reducing the number of features: the feature classes with potentially high number of features (e.g. word bigrams) are reduced to the top $n$ used features, across all authors. The number is chosen to be 50 in most cases, except for bag-of-words and function words, where $\mathrm{n}$ is chosen to be 300 , due to the importance of those features. Table 4.5 shows 22 features that are used in the experiments. The " $\leqslant$ " sign in the dimension column of each row shows the maximum dimension for that feature using the reduction heuristic mentioned above. This is different from the maximum dimension possible for that feature. E.g. if no reduction is applied, there are at most 676 letter bigrams ( $a d, a b, \ldots, z y, z z)$.

There are 22 features for this feature set which can be classified into 4 different categories: Lexical, Syntactic, Content and Idiosyncratic.

Lexical features are the smallest unit of information (characters and words) to be considered. These include style markers that were used in previous work on author identification such as sentence/line length [5] and word-length distributions [17]. We also inspect the usage frequency of word capitalization that would serve as a differentiating component of an author's writing style. For example, some users type very fast which results in the whole contents in lowercase while other carcful uscrs try their best to write clean and readable messages.

Syntactic features are higher level features which represent the writing style of writers. These includes the preferred usage of grammar, tense, voice and punctuation. In addition, syntactic features also include function words which have shown to be highly effective in differentiating between different writing styles [27]. Other syntactic features are part-of-speech tag n-grams.

Content-specific features are important features which reflect the content if different topics [32] such as word n-grams [18]. For example, a discussion in a football forum may contain content-specific features such as "goalkeeper", "referee", "coach", 


\section{Chapter 4. Experiments}

etc. In the case of Dark Web forums, different users will often have different interests. Weapon seekers often mention about "gun", "bullet", "range" while merchants prefer to use some terms such as "for sale", "discount", "offer" etc.

The only idiosyneratic feature in this feature set is misspellings of words. Idiosyncratic features constitute another important subset of features which may help to characterize cultural differences or personal preferences such as "center" vs "centre" [28]. 


\begin{tabular}{|c|c|c|c|}
\hline Group & Feature Name & Dimension & Feature Description \\
\hline Lexical & Character count & 1 & $\begin{array}{l}\text { The total number of characters } \\
\text { in the document }\end{array}$ \\
\hline Lexical & $\begin{array}{l}\text { Average characters } \\
\text { per word }\end{array}$ & 1 & $\begin{array}{l}\text { Average number of characters } \\
\text { per word in the document }\end{array}$ \\
\hline Lexical & Letters & 26 & $\begin{array}{c}\text { Frequency of letters (a-z, case } \\
\text { insensitive) }\end{array}$ \\
\hline Lexical & Top Letter bigrams & $\leqslant 50$ & $\begin{array}{c}\text { Most common letter bigrams } \\
\text { within words (e.g. aa, ab, etc.), } \\
\text { casc insensitive. }\end{array}$ \\
\hline Lexical & Top Letter trigrams & $\leqslant 50$ & $\begin{array}{l}\text { Most common letter trigrams } \\
\text { within words (c.g. aaa, aab, etc.), } \\
\text { case insensitive. }\end{array}$ \\
\hline Lexical & Digits Percentage & 1 & $\begin{array}{c}\text { Percentage of digits out of } \\
\text { the total character count }\end{array}$ \\
\hline Lexical & Letters Percentage & 1 & $\begin{array}{l}\text { Percentage of letters out of } \\
\text { the total character count }\end{array}$ \\
\hline Lexical & $\begin{array}{l}\text { Uppercase Letters } \\
\text { Percentage }\end{array}$ & 1 & $\begin{array}{c}\text { Percentage of uppercase letters } \\
\text { out of the total characters }\end{array}$ \\
\hline Lexical & Digits & 10 & Frequency of digits $(0,1, \ldots, 9)$ \\
\hline Lexical & $\begin{array}{l}\text { Two Digit } \\
\text { Numbers }\end{array}$ & $\leqslant 100$ & $\begin{array}{c}\text { Frequencies of } 2 \text { digit } \\
\text { numbers (e.g. 06, 11, 99, etc.). }\end{array}$ \\
\hline Lexical & $\begin{array}{l}\text { Three Digit } \\
\text { Numbers }\end{array}$ & $\leqslant 1000$ & $\begin{array}{c}\text { Frequencies of } 3 \text { digit } \\
\text { numbers (e.g. 027, 100, etc.). }\end{array}$ \\
\hline Lexical & Word Lengths & N.A. & $\begin{array}{c}\text { Frequency of words of } \\
\text { different lengths }\end{array}$ \\
\hline Lexical & $\begin{array}{c}\text { Special } \\
\text { Characters }\end{array}$ & $\leqslant 21$ & $\begin{array}{c}\text { Frequencies of special } \\
\text { characters, e.g. } \sim \text {, @, etc. }\end{array}$ \\
\hline Syntactic & $\begin{array}{l}\text { Function } \\
\text { Words }\end{array}$ & $\leqslant 50$ & $\begin{array}{l}512 \text { common function words, } \\
\text { used by Koppel et al. (2005) }\end{array}$ \\
\hline Syntactic & Punctuation & 8 & Punctuation symbols \\
\hline Syntactic & POS Tags & $\leqslant 50$ & Part-Of-Speech tags \\
\hline Syntactic & POS Bigrams & $\leqslant 50$ & Part-Of-Speech tag bigrams \\
\hline Syntactic & POS Trigrams & $\leqslant 50$ & Part-Of-Speech tag trigrams \\
\hline Content & Words & $\leqslant 50$ & $\begin{array}{l}\text { Frequencies of various words } \\
\text { in the text }\end{array}$ \\
\hline Content & Word Bigrams & $\leqslant 50$ & $\begin{array}{c}\text { Frequencies of various word } \\
\text { bigrams in the text }\end{array}$ \\
\hline Content & Word Trigrams & $\leqslant 50$ & $\begin{array}{l}\text { Frequencies of various word } \\
\text { trigrams in the text }\end{array}$ \\
\hline Idiosyncratic & Misspelled Words & $\leqslant 50$ & $\begin{array}{l}\text { Frequencies of misspelled } \\
\text { words from a list of } 5,513 \\
\text { common misspellings }\end{array}$ \\
\hline
\end{tabular}

Table 4.5: Fcatures vectors description 


\subsection{Classifier Generation}

The next step is to choose a good classification algorithm to learn from the features extracted from Jstylo for classifying the texts. Many attempts using different learning methods for authorship attribution have been done. The pool of studied techniques includes statistical methords, for example, principal component analysis (PCA), multiple regression, discriminant analysis [42], neural networks [26], colony optimization [39], genetic algorithms [22], Markov models [20], etc. At first, it seems to be uneasy to decide which choice of learning method is the best option for our task. However, studies $[52,53]$ cited in $[44]$ have demonstrated that most classification methods are comparable in performance when the training set is sufficiently large.

We decided to use support vector machine, which is a popular classification technique and has been shown to be highly effective as a tool for authorship attribution and document classification [2]. In addition, empirical experiments have shown that SVM (polynomial and rbf) worked better than other best performing conventional methods (naive Bayes, Rocchio, decision trees, k-nearest neighbor) [18]. In order to avoid reinventing the wheel, we decided to use libSVM [10], an open source SVM library to carry out the experiments. The usage of this library is appropriate in our case as the focus of this thesis is the study of the effectiveness of different features on the classification performance. For both experiments, we divide the dataset into separate training and testing parts.

A data conversion process is required to transform the Jstylo feature output format into the libSVM compatible format. Jstylo output and libSVM input are both stored in the sparse array form. It means that only the non-zero data are stored while any missing data is taken as holding value zero. However, they are different when considering about the starting index. The Jstylo format is zero-based while the libSVM format is one-based. In other words, Jstylo feature index starts at 0 and libSVM feature index starts at 1. Furthermore, their feature representations are also not equivalent. Table 4.6 shows the differences between Jstylo and libSVM feature representations. 


\begin{tabular}{|c|c|c|c|}
\hline & Feature representations & Separation & Example \\
\hline Jstylo & Feature-index Feature-value & Comma (",") & $50,63,74.532$ \\
\hline LibSVM & Feature-index:Feature-value & Space (" ") & $5: 06: 3 \quad 7: 4.532$ \\
\hline
\end{tabular}

Table 4.6: Data representation differences between Jstylo and LibSVM

Another stép in the data conversion process is the removal of redundant data from Jstylo that cannot be understood by libSVM. Jstylo always appends a features description text block which tells the meaning of each feature to the top of the feature file. The feature descriptions help us to understand what each feature index stands for but are irrelevant and not required by libSVM.

Based on the fact that SVM only performs binary classification task, we apply the $\mathrm{N}$ two-way classification model, where $\mathrm{N}$ is equal to the number of testing authors $(N=10)$. As a result, each SVM classification was applied $N$ times on the testing set of documents. This is known as the one-against-all approach. The basic idea of this approach is to view the problem in a different angle than the traditional binary classification task. Rather than interpreting the problem as learning "author 1 versus author $2 \ldots$ versus author n", we can consider it as "learning author 1 versus the rest, author 2 versus the rest, ..., author $\mathrm{n}$ versus the rest". In the end, the $\mathrm{N}$ binary classification problems are equivalent. In geometrical terms, we can consider this problem as scarching for $\mathrm{N}$ separating hyperplanes, each of which represents each individual author's writing style. Figure 4.4 helps to illustrate this approach. 


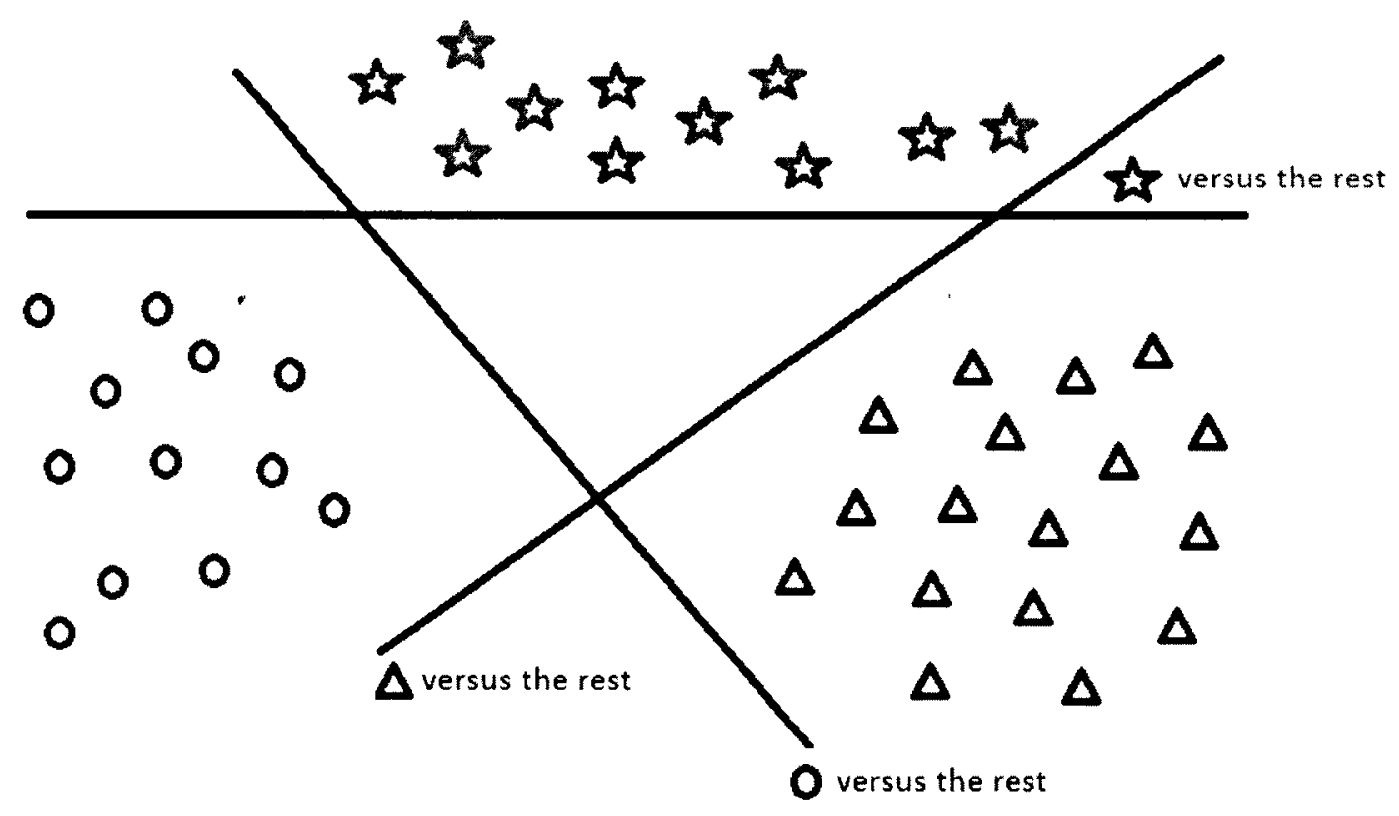

Figure 4.4: Onc-against-all SVM classification

To predict a new instance of the testing set, we choose the classifier with the largest decision function value. The generated results are in the form of confusion matrices, cach matrix's size is $10^{*} 10$. To evaluate the performance of this classification model, we compute the following metrics: recall $(\mathrm{R})$, precision $(\mathrm{P})$ and $F_{1}$ measure. Recall and precision are defined as below:

$$
\begin{gathered}
P_{i}=\frac{T P_{i}}{T P_{i}+F P_{i}} \\
R_{i}=\frac{T P_{i}}{T P_{i}+F N_{i}}
\end{gathered}
$$

There are four combinations of true labels and predicted label. $T P_{i}$ (True Positive) is the percentage of documents that the classifier correctly ascribes to author $i . T N_{i}$ (True Negative) is the percentage of documents that the classifier correctly does not ascribe to author i. FP (False Positive) is the percentage of documents that the classifier wrongly ascribes to author $i . F N_{i}$ (False Positive) is the percentage of documents that the classifier wrongly doesn't ascribe to author $i$. 


\section{Chapter 4. Experiments}

The $F_{1}$ measure is equal to the harmonic mean of recall and precision. Therefore, the $F_{1}$ values are in the interval $(0,1)$. $F_{1}$ measure can holp to determine the performance of the classification model. The larger $F_{1}$ values are equivalent to higher classification quality. There are two different methods of calculating the $F_{1}$ values, which are micro-average and macro-average. Micro-averaged $\mathbf{F}_{1}$ measure: $F_{1}$ is computed globally over all category decisions (authors). P and $\mathrm{R}$ are calculated by the total of all individual decisions as shown in Equation 4.3 and Equation $4.4(\mathrm{~N}$ is the number of testing authors as we defined above).

$$
\begin{aligned}
& P=\frac{T P}{T P+F P}=\frac{\sum_{i=1}^{N} T P_{i}}{\sum_{i=1}^{N}\left(T P_{i}+F P_{i}\right)} \\
& R=\frac{T P}{T P+F N}=\frac{\sum_{i=1}^{N} T P_{i}}{\sum_{i=1}^{N}\left(T P_{i}+F N_{i}\right)}
\end{aligned}
$$

Nicro-averaged $F_{1}$ is then computed as:

$$
F_{1}(\text { micro })=\frac{2 \times R \times P}{R+P}
$$

The problem with micro-averaged $F_{1}$ is that it gives equal weight to each document. As a consequence, authors with more documents tend to dominate authors with less document in the final measure. To remedy this problem, we also computer the macroaveraged $F_{1}$ measure.

Macro-averaged $F_{1}$ measure: We first compute $P$ and $R$ according to Equation 4.3 and 4.4. The global value of $F_{1}$ is then simply the average of local $F_{1}$ values computcd locally for cach author:

$$
\begin{gathered}
F_{1 i}=\frac{2 \times R_{i} \times P_{i}}{R_{i}+P_{i}} \\
F_{i}(\text { macro })=\frac{\sum_{i=1}^{N} F_{1 i}}{N}
\end{gathered}
$$

The purpose of using $F_{1}$ measure is to allow a fair comparison between different authors who have different frequencies of occurrence in the dataset. This property is important because the classifier may be biased against less known authors. 
For the first validation experiment, we apply the classification model using different SVM kernels together with sequential minimal optimization (SMO) algorithm. Basically, a kernel is a similarity function which we provide to a machine learning algorithm. It takes two inputs and estimates how similar they are. We carricd out the experiment independently for each kernel type. The confusion matrices of all experiments are recorded. The row labels of each matrix are the names of the 10 classifiers used in the experiments (training authors) while the column labels are the 10 labeled testing documents. The number in each row $(i, j)$ is the classifying decision value (distance to hyperplane) that the classifier in row $i$ classifies the testing data of author in column $j$. The label (author) of each test document $i$ is the highest value in column $i$ which is highlighted in red color. After that, using the optimal kernel (which leads to the best accuracy), we apply the model on the testing dataset. The following tables (Table 4.7, Table 4.8, Table 4.9) show the results of the validation experiments using different kernels.

\begin{tabular}{|c|c|c|c|c|c|c|c|c|c|c|}
\hline & $\mathbf{B M}(\mathbf{t})$ & $\mathbf{C W}(\mathbf{t})$ & $\mathbf{F T}(\mathbf{t})$ & $\mathbf{K a}(\mathbf{t})$ & $\mathbf{T a}(\mathbf{t})$ & $\mathbf{Z i}(\mathbf{t})$ & $\mathbf{c v}(\mathbf{t})$ & $\mathbf{m a}(\mathbf{t})$ & $\mathbf{m u}(\mathbf{t})$ & $\mathbf{r i}(\mathbf{t})$ \\
\hline $\mathbf{B M}(\mathbf{t r})$ & $0.01 \times$ & -1.154 & -2.3 & -1.91 & -0.541 & -0.776 & 0.777 & -1.695 & -1.467 & -1.554 \\
\hline $\mathbf{C W}(\mathbf{t r})$ & -1.519 & -0.321 & -1.598 & -2.091 & -0.035 & -1.079 & -1.821 & -2.18 & -2.336 & -1.858 \\
\hline $\mathbf{F T}(\mathbf{t r})$ & -1.507 & -0.944 & $-0.10 i$ & -1.235 & -1.634 & -1.08 & -1.272 & -0.639 & -1.005 & -1.084 \\
\hline $\mathbf{K a}(\mathbf{t r})$ & -1.943 & -1.953 & -1.226 & 0.141 & -2.857 & -1.888 & -1.961 & -0.611 & -0.393 & -0.91 \\
\hline $\mathbf{T a}(\mathbf{t r})$ & -2.158 & -2.541 & -3.203 & -3.628 & 1.582 & -3.48 & -2.795 & -3.782 & -3.865 & -3.817 \\
\hline $\mathbf{Z i}(\mathbf{t r})$ & -1.813 & -0.932 & -1.387 & -1.066 & -4.126 & 0.816 & -2.009 & -1.02 & -0.882 & -0.722 \\
\hline $\mathbf{c v}(\mathbf{t r})$ & -0.927 & -0.984 & -1.413 & -1.372 & -0.959 & -0.791 & 1.633 & -1.329 & -1.199 & -1.132 \\
\hline $\mathbf{m a}(\mathbf{t r})$ & -1.64 & -1.469 & -1.082 & -1.063 & -2.783 & -1.314 & -1.633 & 0.531 & -0.801 & -1.382 \\
\hline $\mathbf{m u}(\mathbf{t r})$ & -2.443 & -1.95 & -1.232 & -0.828 & -5.082 & -1.311 & -2.812 & -0.411 & 0.4122 & -1.1 \\
\hline $\mathbf{r i}(\mathbf{t r})$ & -1.624 & -1.381 & -1.485 & -1.093 & -1.734 & -1.197 & -0.709 & -1.545 & -1.289 & 0.544 \\
\hline
\end{tabular}

Table 4.7: Validation experiment result (linear kernel)

\begin{tabular}{|c|c|c|c|c|c|c|c|c|c|c|}
\hline & $\mathbf{B M}(\mathbf{t})$ & $\mathbf{C W}(\mathbf{t})$ & $\mathbf{F T}(\mathrm{t})$ & $\mathbf{K a}(\mathbf{t})$ & $\mathbf{T a}(\mathbf{t})$ & $\mathbf{Z i}(\mathbf{t})$ & $\mathbf{c v}(\mathbf{t})$ & $\mathbf{m a}(\mathbf{t})$ & $\mathbf{m u}(\mathbf{t})$ & $\mathbf{r i}(\mathbf{t})$ \\
\hline $\mathbf{B M}(\mathbf{t r})$ & 0.103 & -1.16 & -2.256 & -1.85 & -0.291 & -0.79 & 0.924 & -1.614 & -1.423 & -1.535 \\
\hline $\mathbf{C W}(\mathbf{t r})$ & -1.422 & -0.116 & -1.519 & -1.986 & -0.132 & -1.134 & -1.549 & -2.043 & -2.15 & -1.742 \\
\hline $\mathbf{F T}(\mathbf{t r})$ & -1.589 & -0.938 & -0.37 & -1.238 & -2.058 & -1.082 & -1.309 & -0.675 & -1.007 & -1.086 \\
\hline $\mathbf{K a}(\mathbf{t r})$ & -2.083 & -2.071 & -1.229 & 0.127 & -3.721 & -1.918 & -2.081 & -0.704 & -0.431 & -0.927 \\
\hline $\mathbf{T a}(\mathbf{t r})$ & -2.059 & -2.365 & -2.865 & -3.157 & 1.696 & -3.043 & -2.559 & -3.298 & -3.318 & -3.281 \\
\hline $\mathbf{Z i}(\mathbf{t r})$ & -1.912 & -0.89 & -1.418 & -1.109 & -5.624 & $0 . \times 6$ & -2.018 & -1.091 & -0.898 & -0.749 \\
\hline $\mathbf{c v}(\mathbf{t r})$ & -0.914 & -0.98 & -1.374 & -1.346 & -0.954 & -0.832 & 1.65 & -1.296 & -1.191 & -1.141 \\
\hline $\mathbf{m a}(\mathbf{t r})$ & -1.757 & -1.518 & -1.067 & -1.061 & -3.709 & -1.34 & -1.752 & $0.7 \times$ & -0.794 & -1.424 \\
\hline $\mathbf{m u}(\mathbf{t r})$ & -2.703 & -2.066 & -1.198 & -0.851 & -7.206 & -1.34 & -3.192 & -0.447 & 0.176 & -1.093 \\
\hline $\mathbf{r i}(\mathbf{t r})$ & -1.732 & -1.411 & -1.478 & -1.11 & -2.259 & -1.208 & -0.678 & -1.537 & -1.285 & 0.653 \\
\hline
\end{tabular}

Table 4.8: Validation experiment result (polynomial kernel) 


\begin{tabular}{|c|c|c|c|c|c|c|c|c|c|c|}
\hline & $\mathbf{B M}(\mathbf{t})$ & $\mathbf{C W}(\mathbf{t})$ & $\mathbf{F T}(\mathbf{t})$ & $\mathbf{K a}(\mathbf{t})$ & $\mathbf{T a}(\mathbf{t})$ & $\mathbf{Z i}(\mathbf{t})$ & $\mathbf{c v}(\mathbf{t})$ & $\mathbf{m a}(\mathbf{t})$ & $\mathbf{m u}(\mathbf{t})$ & $\mathbf{r i}(\mathbf{t})$ \\
\hline $\mathbf{B M}(\mathbf{t r})$ & -0.952 & -0.995 & -1.105 & -1.061 & -0.998 & -0.986 & -0.988 & -1.003 & -1.018 & -1.031 \\
\hline $\mathbf{C W}(\mathbf{t r})$ & -1.02 & -1.003 & -1.001 & -1.001 & -0.989 & -1 & -0.989 & -0.995 & -0.999 & -0.995 \\
\hline $\mathbf{F T}(\mathbf{t r})$ & -1 & -1.019 & -0.825 & -0.976 & -0.987 & -1.015 & -0.988 & -0.971 & -1.003 & -0.954 \\
\hline $\mathbf{K a}(\mathbf{t r})$ & -1.038 & -1.056 & -1.019 & -0.71 & -1.033 & -1.062 & -1.033 & -0.986 & -0.91 & -0.981 \\
\hline $\mathbf{T a}(\mathbf{t r})$ & -0.997 & -1.008 & -1.003 & -1.003 & -0.61 & -0.999 & -0.952 & -0.977 & -0.995 & -0.979 \\
\hline $\mathbf{Z i}(\mathbf{t r})$ & -1.09 & -1.091 & -1.011 & -0.956 & -1.082 & -0.70 & -1.083 & -1.068 & -1.057 & -1.012 \\
\hline $\mathbf{c v}(\mathbf{t r})$ & -0.936 & -1.962 & -1.015 & -1 & -0.963 & -0.997 & -0.423 & -0.983 & -0.995 & -0.984 \\
\hline $\mathbf{m a}(\mathbf{t r})$ & -0.998 & -1.006 & -1.008 & -1.006 & -0.96 & -1.008 & -0.961 & -0.37 .1 & -0.954 & -0.991 \\
\hline $\mathbf{m u}(\mathbf{t r})$ & -1.021 & -1.033 & -1.069 & -0.905 & -1.019 & -1.067 & -1.02 & -0.91 & -0.656 & -1.009 \\
\hline $\mathbf{r i}(\mathbf{t r})$ & -1.004 & -1.041 & -1.002 & -0.947 & -0.977 & -1.036 & -0.978 & -1.007 & -1.02 & -0.39 .3 \\
\hline
\end{tabular}

Table 4.9: Validation experiment result (rbf kernel)

From the above results, we can observe that the linear and polynomial kernels can achieve 10/10 correct classifications. This result agrees with numerous previous studies [3] as the combination of linear kernel and SMO is the most used parameter setting. Table 4.10 shows the $F_{1}$ scores of the validation experiments.

\begin{tabular}{lll}
\hline Kernel & $F_{1}$ macro & $F_{1}$ micro \\
\hline Lincar & 1 & 1 \\
Polynomial & 1 & 1 \\
Rbf & 0.765 & 0.8
\end{tabular}

Table 4.10: Validation experiments $F_{1}$ score

In the next step, we carried out the test experiment using linear and polynomial kernels which achicved the perfect classification results for the first experiments. The results of this testing phase are recorded in Table 4.11 and Table 4.12. Table 4.13 shows the $F_{1}$ scores of the test experiments.

\begin{tabular}{|c|c|c|c|c|c|c|c|c|c|c|}
\hline & $\mathrm{BM}(\mathrm{t})$ & $\mathrm{CW}(\mathbf{t})$ & $\mathbf{F T}(\mathrm{t})$ & $\mathrm{Ka}(\mathrm{t})$ & $\mathrm{Ta}(\mathrm{t})$ & $\mathrm{Zi}(\mathrm{t})$ & $\operatorname{cv}(t)$ & $\mathrm{ma}(\mathrm{t})$ & $m u(t)$ & $\mathrm{ri}(\mathrm{t})$ \\
\hline $\mathrm{BM}(\mathrm{tr})$ & $-0.07: 3$ & -0.62 & -2.023 & -1.841 & -1.201 & -1.036 & -0.328 & -1.61 & -1.177 & -1.952 \\
\hline CW(tr) & -1.678 & -0.686 & -1.635 & -2.254 & -3.024 & -1.48 & -1.831 & -2.608 & -2.754 & -2.58 \\
\hline FT(tr) & -1.39 & -0.995 & -0.662 & -1.144 & -1.468 & -1.094 & -1.14 & -0.932 & -1.169 & -0.903 \\
\hline Ka(tr) & -2.119 & -2.084 & -1.632 & 9). 161 & -1.258 & -1.793 & -2.333 & -0.519 & -0.492 & -0.649 \\
\hline $\mathrm{Ta}(\mathrm{tr})$ & -2.649 & -3.012 & -2.947 & -3.417 & -4.152 & -3.083 & -4.113 & -4.027 & -4.216 & -4.571 \\
\hline $\mathrm{Zi}(\mathrm{tr})$ & -1.326 & -0.36 & -1.64 & -1.532 & -1.642 & -0.066 & -0.758 & -0.967 & -0.559 & -0.551 \\
\hline $\operatorname{cv}(\mathrm{tr})$ & -1.004 & -0.683 & -1.585 & -1.271 & -1.306 & -1.04 & 0.188 & -1.273 & -1.257 & -1.371 \\
\hline $\operatorname{ma}(t r)$ & -1.588 & -1.353 & -1.324 & -1.134 & -1.411 & -1.327 & -1.342 & -0.327 & -0.946 & -1.078 \\
\hline $\mathrm{mu}(\mathrm{tr})$ & -2.019 & -2.043 & -1.678 & -0.899 & -2.096 & -1.681 & -1.369 & -0.616 & 0.831 & -0.372 \\
\hline$r i(t r)$ & -1.703 & -1.419 & -1.563 & -1.011 & -1.26 & -1.27 & -1.042 & -1.415 & -1.286 & 0.173 \\
\hline
\end{tabular}

Table 4.11: Test experiment result (linear kemel) 


\begin{tabular}{|c|c|c|c|c|c|c|c|c|c|c|}
\hline & $\mathbf{B M}(\mathbf{t})$ & $\mathbf{C W}(\mathbf{t})$ & $\mathbf{F T}(\mathrm{t})$ & $\mathbf{K a}(\mathbf{t})$ & $\mathbf{T a}(\mathbf{t})$ & $\mathbf{Z i}(\mathbf{t})$ & $\mathbf{c v}(\mathrm{t})$ & $\mathbf{m a}(\mathbf{t})$ & $\mathbf{m u}(\mathbf{t})$ & $\mathbf{r i}(\mathbf{t})$ \\
\hline $\mathbf{B M}(\mathbf{t r})$ & $-1) .019$ & -0.623 & -2.008 & -1.801 & -1.206 & -1.042 & -0.421 & -1.55 & -1.167 & -1.824 \\
\hline $\mathbf{C W}(\mathbf{t r})$ & -1.546 & -0.777 & -1.518 & -2.072 & -2.583 & -1.425 & -1.778 & -2.341 & -2.451 & -2.363 \\
\hline $\mathbf{F T}(\mathbf{t r})$ & -1.429 & -0.994 & -0.614 & -1.151 & -1.455 & -1.099 & -1.138 & -0.944 & -1.163 & -0.925 \\
\hline $\mathbf{K a}(\mathbf{t r})$ & -2.224 & -2.162 & -1.67 & 0.176 & -1.238 & -1.849 & -2.28 & -0.582 & -0.551 & -0.723 \\
\hline $\mathbf{T a}(\mathbf{t r})$ & -2.455 & -2.724 & -2.679 & -3.013 & -3.532 & -2.77 & -3.486 & -3.436 & -3.538 & -3.764 \\
\hline $\mathbf{Z i}(\mathbf{t r})$ & -1.334 & -1.294 & -1.701 & -1.558 & -1.625 & 0.0112 & -0.762 & -1.013 & -0.611 & -0.656 \\
\hline $\mathbf{c v}(\mathbf{t r})$ & -1.005 & -0.715 & -1.535 & -1.262 & -1.315 & -1.046 & -0.075 & -1.259 & -1.249 & -1.349 \\
\hline $\mathbf{m a}(\mathbf{t r})$ & -1.683 & -1.382 & -1.353 & -1.138 & -1.45 & -1.357 & -1.38 & -0.363 & -0.968 & -1.103 \\
\hline $\mathbf{m u}(\mathbf{t r})$ & -2.15 & -2.158 & -1.707 & -0.916 & -2.139 & -1.729 & -1.44 & -0.648 & 0.711 & -0.447 \\
\hline $\mathbf{r i}(\mathbf{t r})$ & -1.779 & -1.441 & -1.574 & -1.026 & -1.314 & -1.285 & -1.077 & -1.413 & -1.287 & 0.255 \\
\hline
\end{tabular}

Table 4.12: Test experiment result (polynomial kernel)

\begin{tabular}{lll}
\hline Kernel & $F_{1}$ macro & $F_{1}$ micro \\
\hline Linear & 0.747 & 0.8 \\
Polynomial & 0.747 & 0.8
\end{tabular}

Table 4.13: Test experiments $F_{1}$ score

The results show that there is not much difference between the performance of linear and polynomial kernels. Both kernels can achieve $80 \%$ accuracy $(8 / 10$ correct classifications) which is a quite good result in authorship attribution domain. Table 4.14 and 4.15 compare the results of the above SVM classifications with the other methods which result in a slightly worse performance than SVM.

\begin{tabular}{|c|c|c|}
\hline & Validation Experiment & Testing Experiment \\
\hline SVM & 1 & 0.8 \\
\hline Random Forest & 0.9 & 0.8 \\
\hline XGBoost & 0.7 & 0.6 \\
\hline
\end{tabular}

Table 4.14: Experiment results using different classification methods in micro $F_{1}$

\begin{tabular}{|c|c|c|}
\hline & Validation Experinent & Testing Experiment \\
\hline SVM & 1 & 0.747 \\
\hline Random Forest & 0.874 & 0.747 \\
\hline XGBoost & 0.616 & 0.530 \\
\hline
\end{tabular}

Table 4.15: Expcriment results using different classification methods in macro $F_{1}$ 


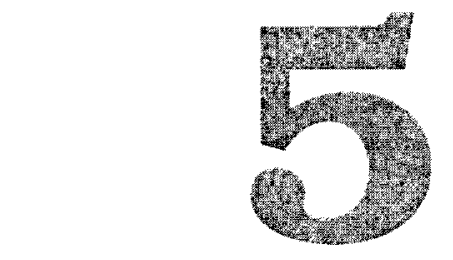

Conclusion

In this research, we have built a framework for authorship matching based on features extracted from online messages in Dark Web forums. We have undertaken two separate experiments to evaluate the effectiveness of the proposed method. These experiments have shown that writing style can be used to attribute and correlate authorship between users on multiple Dark Web forums with high accuracy. We believe that the proposed framework has the potential to aid the task of tracing secret cyber criminal identities that are hidden under the indexed surface web.

However, there are some constraints associated with this research. Firstly, we only consider stylometric attributes that work at the character level, word level, sentence level. and document level. There are some other attributes that work at the phrase level, the clause level, and the paragraph level. These attributes, however, are not considered in this study. Given their properties, we can assume that these attributes should provide good results at least for the text summarization task. Secondly, as mentioned in previous chapters, we assume that no text obfuscation attempts are made by the users. The problem can be complicated if the users intentionally alter their writing style to avoid being undercovered by legal partics. Therefore, sophisticated techniques for detecting stylistic deception in written texts need to be integrated 
for handling such complex scenarios. Last but not least, the size of our test dataset (users who have at least two active accounts on different Dark Web forums) is small due to the limitations of the archived data that we use. We can overcome this problem by creating a data crawler to collect more data dircetly from Dark Wcb forums which can potentially help to increase the dimension of our test dataset.

In addition to the limitations of this research that we need to overcome, we have identified several promising research ideas based on the current study. There are three directions that can be considered as future works for this thesis.

(i) Firstly, as this study only focused on English messages, we plan to include more languages into our future rescarches.

(ii) Secondly, we plan to investigate the feasibility of authorship matching based on online messages written in other languages, both alphabetical and nonalphabetical ones.

(iii) Finally, we will try to focus more on other features that are less related to writing style such as topic, posted date/location, users forum signature, semantic features, etc. and apply this technique to find the corrclations of uscrs between dark web and social networks. 


\section{List of Authors Publications}

\subsection{Accepted Paper}

(i) Ho, Thanh Nghia, and Wee Keong Ng. "Application of Stylometry to DarkWeb Forum User Identification." Information and Communications Security. Springer International Publishing, 2016. 173-183. 


\section{References}

[1] Ahmed Abbasi and Hsinchun Chen. Applying authorship analysis to arabic web content. In Intelligence und Security Informatics, pages 183-197. Springer, 2005.

[2] Ahmed Abbasi and Hsinchun Chen. Applying authorship analysis to extremistgroup web forum messages. Intelligent Systems, IEEE, 20(5):67-75, 2005.

[3] Ahmed Abbasi and Hsinchun Chen. Writeprints: A stylometric approach to identity-level identification and similarity detection in cyberspace. ACM Transactions on Information Systems (TOIS), 26(2):7, 2008.

[4] Mishari Almishari, Paolo Gasti, Gene Tsudik, and Ekin Oguz. Privacy-preserving matching of community-contributed content. In European Symposium on Research in Computer Security, pages 443-462. Springer, 2013.

[5] Shlomo Argamon, Marin Sarić, and Sterling S Stcin. Style mining of elcctronic messages for multiple authorship discrimination: first results. In Proceedings of the ninth ACM SIGKDD international conference on Knowledge discovery and data mining, pages 475-480. ACM, 2003.

[6] Harald Baayen, Hans van Halteren, Anneke Neijt, and Fiona Tweedie. An experiment in authorship attribution. In 6th JADT, pages 29-37, 2002.

[7] Michael Robert Brennan and Rachel Greenstadt. Practical attacks against authorship recognition techniques. In $I A A I, 2009$.

[8] John F Burrows. Word-patterns and story-shapes: The statistical analysis of narrative style. Literary and linguistic Computing, 2(2):61-70, 1987. 


\section{REFERENCES}

[9] Aylin Caliskan-Islam, Richard Harang, Andrew Liu, Arvind Narayanan, Clare Voss, Fabian Yamaguchi, and Rachel Greenstadt. De-anonymizing programmers via code stylometry. In 24th USENIX Security Symposium (USENIX Security 15), pages $255-270,2015$.

[10] Chih-Chung Chang and Chih-Jen Lin. Libsrm: a library for support vector machines. ACM Transactions on Intelligent Systems and Technology (TIST), $2(3): 27,2011$.

[11] Carole Chaski. Variables and method for authorship attribution, April 62006. US Patent App. 11/398,728.

[12] Carole E Chaski. Who's at the keyboard? authorship attribution in digital evidence investigations?, int. L J. DIGITAL EVIDENCE, Spring, pages 9-10, 2005.

[13] Marco Cristani, Giorgio Roffo, Cristina Segalin, Loris Bazzani, Alessandro Vinciarelli, and Vittorio Murino. Conversationally-inspired stylometric features for authorship attribution in instant messaging. In Proceedings of the 20th ACM international conference on Multimedia, pages 1121-1124. ACM, 2012.

[14] Nello Cristianini and John Shawe-Taylor. An introduction to support vector machines and other kernel-based learning methods. Cambridge university press, 2000.

[15] DailyMail. Teen was hours away from columbine-style massacre at his old school: Ex-student, 19. 'stockpiled weapons and explosives including a 9mm pistol and five pipe bombs in a bid to carry out mass murder, July 2015.

[16] Olivier De Vel. Mining e-mail authorship. In Proc. Workshop on Text Mining, ACM International Conference on Knowledge Discovery and Data Mining (KDD2000), 2000. 


\section{REFERENCES}

[17] Olivier De Vel, Alison Anderson, Malcolm Corney, and George Mohay. Mining e-mail content for author identification forensics. ACM Sigmod Record, 30(4):55$64,2001$.

[18] Joachim Diederich, Jörg Kindermann, Edda Leopold, and Gerhard Paass. Authorship attribution with support vector machines. Applied intelligence, 19(12):109-123, 2003.

[19] Macicj Edcr. Does sizc matter? authorship attribution, small samples, big problem. Digital Scholarship in the Humanities, page fqt066, 2013.

[20] Erik Goldman and Abel Allison. Using grammatical markov models for stylometric analysis.

[21] David I Holmes. A stylometric analysis of mormon scripture and related texts. Journal of the Royal Statistical Society. Series A (Statistics in Society), pages 91-120, 1992.

[22] David I Holmes and Richard S Forsyth. The federalist revisited: New directions in authorship attribution. Literary and Linguistic Computing, 10(2):111-127, 1995.

[23] Anil Jain, Arun A Ross, and Karthik Nandakumar. Introduction to biometrics. Springer Science \& Business Media, 2011.

[24] Patrick Juola. Authorship attribution. Foundations and Trends in information Retrieval, 1(3):233-334, 2006.

[25] Gary Kacmarcik and Michael Gamon. Obfuscating document stylometry to preserve author anonymity. In Proceedings of the COLING/ACL on Main conference poster sessions, pages 444 451. Association for Computational Linguistics, 2006.

[26] Bradley Kjell. Authorship determination using letter pair frequency features with neural network classifiers. Literary and Linguistic Computing, 9(2):119$124,1994$. 


\section{REFERENCES}

[27] Moshe Koppel, Navot Akiva, and Ido Dagan. Feature instability as a criterion for selecting potential style markers. Journal of the American Society for Information Science and Technology, 57(11):1519-1525, 2006.

[28] Moshe Koppel and Jonathan Schler. Exploiting stylistic idiosyncrasies for authorship attribution. In Proceedings of IJCAI'03 Workshop on Computational Approaches to Style Analysis and Synthesis, volume 69, page 72, 2003.

[29] Noshe Koppel, Jonathan Schler, and Shlomo Argamon. Computational methods in authorship attribution. Journal of the American Society for information Science and Technology, 60(1):9-26, 2009.

[30] Moshe Koppel and Yaron Winter. Determining if two documents are written by the same author. Journal of the Association for Information Science and Technology, 65(1):178-187, 2014.

[31] R Lipmman. An introduction to computing with neural networks. IEEE ASSP Magazine, 3(4):987.

[32] Colin Martindale and Dean McKenzic. On the utility of content analysis in author attribution: The federalist. Computers and the Humanities, 29(4):259-270, 1995.

[33] Andrew WE McDonald, Sadia Afroz, Aylin Caliskan, Ariel Stolerman, and Rachel Grecnstadt. Use fewer instances of the letter i: Toward writing style anonymization. In Privacy Enhancing Technologies, pages 299-318. Springer, 2012.

[34] Thomas Corwin Mendenhall. The characteristic curves of composition. Science, pages $237-249,1887$.

[35] Thomas Corwin Mendenhall. A mechanical solution of a literary problem. 1903.

[36] Frederick Mosteller and David Wallace. Inference and disputed authorship: The federalist. 1964. 


\section{REFERENCES}

[37] Frederick Mosteller and David L Wallace. Applied Bayesian and classical inference: the case of the Federalist papers. Springer Science \& Business Media, 2012 .

[38] Satoshi Nakamoto. Bitcoin: A peer-to-peer electronic cash system, 2008.

[39] Michacl Oakes. Ant colony optimisation for stylometry: The federalist papers. In Proceedings of the 5th International Conference on Recent Advances in Soft Computing, pages 86-91, 2004.

[40] J. Ross Quinlan. Induction of decision trecs. Machine learning, 1(1):81-106, 1986.

[41] Giorgio Roffo, Marco Cristani, Loris Bazzani, Ha Minh, and Vittorio Murino. Trusting skype: Learning the way people chat for fast user recognition and verification. In Proceedings of the IEEE International Conference on Computer Vision Workshops, pages 748-754, 2013.

[42] Efstathios Stamatatos, Nikos Fakotakis, and Gcorge Kokkinakis. Automatic text categorization in terms of genre and author. Computational linguistics, 26(4):471495,2000 .

[43] Efstathios Stamatatos, Nikos Fakotakis, and George Kokkinakis. Text genre detection using common word frequencies. In Proceedings of the 18 th conference on Computational linguistics-Volume 2, pages 808-814. Association for Computational Linguistics, 2000.

[44] Efstathios Stamatatos, Nikos Fakotakis, and Georgios Kokkinakis. Computerbased authorship attribution without lexical measures. Computers and the $\mathrm{Hu}$ manities, 35(2):193-214, 2001.

[45] Gui-Fa Teng, Mao-Sheng Lai, Jian-Bin Ma, and Ying Li. E-mail authorship mining based on sum for computer forensic. In Machine Learning and Cybernetics, 2004. Proceedings of 2004 International Conference on, volume 2, pages 1204-1207. IEEE, 2004. 


\section{REFERENCES}

[46] RN Totty, RA Hardcastle, and J Pearson. Forensic linguistics: The determination of authorship from habits of style. Journal of the Forensic Science Society, $27(1): 13-28,1987$.

[47] USAToday. Is that painting real? ask a mathematician, May 2007.

[48] Peter Van Kranenburg and Eric Backer. Musical style recognition-a quantitative approach. In Procedings of the Conference on Interdisciplinary Musicology (CIM), pages 106-107, 2004.

[49] Vladimir Vapnik. The nature of statistical learning theory. Springer Science \& Business Media, 2013.

[50] Richard Y Wang, Veda C Storey, and Christopher P Firth. A framework for analysis of data quality research. Knowledge and Data Engineering, IEEE Transactions on, $7(4): 623-640,1995$

[51] Wikipedia. Stylometry - wikipedia, the free encyclopedia, 2016. [Online; accessed 21-May-2016].

[52] Yiming Yang. An evaluation of statistical approaches to text categorization. Information retrieval, 1(1-2):69-90, 1999.

[53] Yiming Yang and Xin Liu. A rc-examination of text catcgorization methods. In Proceedings of the 22nd annual international ACM SIGIR conference on Research and development in information retrieval, pages 42-49. ACM, 1999.

[54] C Udny Yule. The statistical study of literary vocabulary. Cambridge University Press, 2014.

[55] G Udny Yule. On sentence-length as a statistical characteristic of style in prose: With application to two cases of disputed authorship. Biometrika, 30(3/4):363390, 1939. 


\section{REFERENCES}

[56] Yulei Zhang, Shuo Zeng, Li Fan, Yan Dang, Catherine A Larson, and Hsinchun Chen. Dark web forums portal: searching and analyzing jihadist forums. In Intelligence and Security Informatics, 2009. ISI'09. IEEE International Conference on, pages 71-76. IEEE, 2009.

[57] Rong Zheng, Jiexun Li, Hsinchun Chen, and Zan Huang. A framework for authorship identification of online messages: Writing-style features and classification techniques. Journal of the American Socicty for Information Science and Technology, 57(3):378-393, 2006.

[58] Rong Zheng, Yi Qin, Zan Huang, and Hsinchun Chen. Authorship analysis in cybcrcrime investigation. In Intelligence and Security Informatics, pages 59-73. Springer, 2003. 